\title{
Microwave and fusion techniques for the synthesis of mesoporous zeolitic composite adsorbents from bagasse fly ash: sorption of $p$ - nitroaniline and nitrobenzene
}

\author{
Olutayo A. Oluyinka ${ }^{1} \cdot$ Alpesh V. Patel ${ }^{2} \cdot$ Bhavna A. Shah $^{1} \cdot$ Maryam I. Bagia $^{1}$
}

Received: 5 October 2019 / Accepted: 28 October 2020 / Published online: 9 November 2020

(c) The Author(s) 2020

\begin{abstract}
$p$-Nitroaniline (PNAN) and nitrobenzene (NB) being important raw materials and intermediates for the production of a wide range of chemical products have the potential of constituting water pollutants. Hence, the development of the means of removing these chemicals from water would go a long way to safeguard the health of the environment. The goal of this research was to transform bagasse fly ash (BFA), solid waste from the sugar industry into porous zeolitic adsorbents MgFZBFA and MgMZBFA and examine them for their adsorptive removal of PNAN and NB from aqueous solutions. The syntheses of the sorbents involve alkali fusion technique and microwave hydrothermal treatment for the desired properties. Advanced characterization techniques such as FTIR (Fourier transform infrared spectroscopy), XRF (X-ray fluorescence spectroscopy), XRD (X-ray diffraction spectroscopy), SEM (scanning electron microscopy), Brunauer-Emmett-Teller (BET)/BarrettJoyner-Halenda (BJH) Method, and TGA (thermogravimetric analysis) were used for the characterization and evaluation of the sorbents' properties. The adsorptive removal of PNAN and NB from aqueous solutions by MgFZBFA and MgMZBFA were investigated. Various parameters such as $\mathrm{pH}$, adsorbent dosage, initial sorbate concentration were optimized during the adsorption experiment to achieve best performance. Adsorption isotherm, kinetics and dynamics were studied. It was found that Langmuir adsorption isotherm model better represents the adsorption processes and that the processes follow pseudosecond-order kinetics. More so, the sorption processes were most possibly completed by both surface sorption (liquid-film diffusion) and intra-particle diffusion. The maximum sorption capacities observed with MgFZBFA for PNAN and NB are $30.86 \mathrm{mg} \mathrm{g}^{-1}$ and $19.92 \mathrm{mg} \mathrm{g}^{-1}$, while with MgMZBFA the values are $12.72 \mathrm{mg} \mathrm{g}^{-1}$ and $10.20 \mathrm{mg} \mathrm{g}^{-1}$, respectively. The performances of MgFZBFA and MgMZBFA for the sorption of PNAN and NB were compared with some adsorbents previously studied for the same purpose, and results show that the adsorbents in the present study exhibit better performances. The application as materials of cheap source for the removal of PNAN and NB from contaminated water could be considered.
\end{abstract}

Keywords Water pollution $\cdot p$-Nitroaniline $\cdot$ Nitrobenzene $\cdot$ Adsorption $\cdot$ Bagasse fly ash

\section{Introduction}

p-Nitroaniline (PNAN) and nitrobenzene (NB) are important raw materials and intermediates for the production of a wide range of chemical products such as antioxidants, antiozonants, gasoline gum inhibitor, pesticides, soaps, shoe polishes, dyes,

Olutayo A. Oluyinka

biodunolu@gmail.com

1 Department of Chemistry, Veer Narmad South Gujarat University, Surat, Gujarat, India

2 Department of Chemistry, Sheth P. T. Mahila College of Arts and Home Science, Vanita Vishram, Surat, Gujarat, India pharmaceuticals, etc. (Nair et al. 1986; Nishino and Spain 1993; Bhatkhande et al. 2004; Qin et al. 2007; Sun et al. 2007; Pan and Guan 2010). They enter into the aquatic environment due to inadequate treatment of waste stream from industries. This causes water pollution and constitute a significant threat to human as well as animal lives. These chemicals have the potential of causing different kinds of ailments such as methemoglobinemia, cancer and damage to vital organs of the body (such as kidney, liver and spleen) and to the central nervous system (EPA Nitrobenzene 2018; NJDEP 2018; PubChem Nitrobenzene 2018; Zheng et al. 2007; Li et al. 2010; Lai et al. 2018). Therefore, adequate treatment of water contaminated with these chemicals is of utmost importance in order to 
minimize the health risks associated with water consumption and usage.

Studies on natural zeolites being used as adsorbents for wastewater treatment have attracted great attention due to their porous nature which gives them the potential to entrap water pollutants (Wang and Peng 2010). Each of the $\mathrm{AlO}_{4}{ }^{5-}$ and $\mathrm{SiO}_{4}{ }^{4-}$ units in a typical zeolite structure are tetrahedrally arranged and bonded together in a way that each $\mathrm{Si}$ and $\mathrm{Al}$ occupy central positions of the tetrahedral arrangements. Each tetrahedra unit consists of four oxygen atoms and one oxygen atom located at corners of each tetrahedron are shared with adjacent tetrahedral crystals. The $\mathrm{T}-\mathrm{O}-\mathrm{T}$ links from the $\mathrm{Si}-\mathrm{O} /$ $\mathrm{Al}-\mathrm{O}$ bonds form a variety of three-dimensional rings which are responsible for the presence of open hollows in the form of cavities and cages (Moshoeshoe et al. 2017). In addition, the presence of triply charged aluminium in the tetrahedra arrangements results in the generation of a net negative charge in the framework which creates an avenue for the framework to be balanced by metal ions. Such metal ions could generate strong local electric fields and attract the negative centers of polar molecules to the surface of a zeolite (Xu et al. 2007). These characteristics further give natural zeolites the great potential of being applied as adsorbents for environmental pollutants. However, the application of synthetic zeolites instead of the natural zeolites could limit the stress on the natural deposits and the critical shortage could be averted (Shah et al. 2019).

Bagasse fly ash is a solid waste generated from the sugar industry and available in ample of amount with negligible cost. Also, it contains high amount of silica which is the primary building block of zeolitic materials. The goal of this research is to transform bagasse fly ash to zeolitic sorbents via alkali fusion method followed by microwave crystallization, and via direct microwave hydrothermal method, which are then studied for their adsorptive removal of PNAN and NB from aqueous solutions.

Fly ash disposal in the environment has been worrisome (Martison 2014; Huang and Chuieh 2015; Raleigh 2016). Hence, it is being reused in order to reduce the frequency of its disposal and the threats it constitutes. It is being reused in several ways such as being applied as constituents of building construction materials (such as cements and blocks), fireproofs, ceramics, and many more (Querol et al. 2002; James and Pandian 2017; Rauf et al. 2017). The outcome of this research could suggest additional ways to put fly ash into use, and more importantly, serving as a cheap raw material for mitigating water pollution.

\section{Materials and methods}

\section{Materials}

Bagasse fly ash (BFA) obtained from Shree Khedut Sahkari Khand Udhyog Mandali Ltd., Bardoli, India, served as the raw material for the synthesis of the zeolitic composite adsorbents: MgFZBFA; MgMZBFA. $p$-Nitroaniline (PNAN: Sigma $\geq 99 \%$ ), and nitrobenzene (NB: Sigma $\geq 99 \%$ ) were used for the preparation of the synthetic adsorbate solution. Sodium hydroxide ( $\geq 99 \%)$, magnesium chloride ( $\geq 98 \%$ ), sodium aluminate ( $\mathrm{Al}$ as $\mathrm{Al}_{2} \mathrm{O}_{3}: 50-56 \%, \mathrm{Na}$ as $\mathrm{Na}_{2} \mathrm{O}: 37-45 \%$ ), and hydrochloric acid $(37 \%)$ were used during syntheses and adsorption procedures. The chemicals were all supplied by SigmaAldrich (USA) and Loba Chemie (India) and used without further purification.

\section{Instruments}

Thermo Nicolet EV300 UV-visible spectrophotometer was used to quantify the residual PNAN and NB in the solutions of the adsorbates at adsorption equilibrium. A pH meter (Equip-Tronics EQ-621) was utilized for $\mathrm{pH}$ measurements. Compositions of the BFA and the synthesized zeolitic composite adsorbents MgFZBFA, and MgMZBFA were investigated using PANalytical AxiosMAX wavelength-dispersive X-ray fluorescence spectrometer. Rigaku MiniFlex XRD BD111915 with $\mathrm{CuK} \alpha$ radiation $(1.542 \AA$ ) was used to obtain the X-ray diffractograms of BFA and the synthesized adsorbents from which their respective constituent mineral phases were identified. The morphologies of the synthesized sorbents were examined with FEI Field Emission Gun Nano Nova FEG-SEM 450 (with EDAX) scanning electron microscope, and their surface functional groups were examined using a PerkinElmer Spectrum GX FTIR spectrometer. Surface area and porosity measurements were obtained using Micromeritics ASAP 2010 BET Porosimeter. And thermal analyses were carried out using Exstar SII 6300 Thermogravimetric/Differential Thermal Analyzer (TG/DTA).

\section{Preparation of contaminated waters}

A $2.0 \mathrm{~L}$ stock solution of each of the water pollutants: PNAN; and NB were prepared. A $500 \mathrm{mg} \mathrm{L}^{-1}$ of PNAN was prepared by dissolving $1.0 \mathrm{~g}$ of PNAN into an appropriate volume of distilled water. And $1000 \mathrm{mg} \mathrm{L}^{-1}$ of NB was prepared by diluting $1.7 \mathrm{~mL}$ of $\mathrm{NB}$ with distilled water to the appropriate volume. The resultant stock solutions were preserved in clean dark Winchester bottles. Lower concentrations of the solutions of the adsorbates were made from 
the stock solutions and utilized as the working solutions for adsorption experiments.

\section{Standard calibration of UV-visible spectrometer for PNAN and NB quantification}

Full-length UV spectra of PNAN and NB were scanned to obtain the $\lambda_{\max }$ of $380 \mathrm{~nm}$ and $260 \mathrm{~nm}$, respectively. Several known concentrations of PNAN and NB were used for the calibrations. Calibration plots of perfectly straight lines (Fig. 1) of absorbance against the concentrations of the adsorbates were obtained up $40 \mathrm{mg} \mathrm{L}^{-1}$. The calibration plots were used for quantification of the adsorptives or adsorbates throughout the adsorption experiments. Dilutions were done for measurements beyond the calibration limits and the dilution factors were used to get the actual concentrations of the adsorbates.

\section{Pretreatment of BFA}

BFA was screened through $90-\mu \mathrm{m}$ sieve and washed with distilled water to remove foreign dirt, floating residue, and soluble impurities. It was then dried at $373 \pm 1 \mathrm{~K}$, allowed to cool to ambient temperature and kept in a clean container for synthesis of zeolitic materials.

\section{Syntheses of the sorbents}

Alkali fusion method was applied for the synthesis of MgFZBFA. A predetermined mixture of the prepared BFA, sodium aluminate, magnesium chloride and sodium hydroxide were thoroughly ground and mixed. Fly ash to sodium aluminate to magnesium chloride mass ratios were 1: $0.5: 0.5$, while the ratio of their combined mass to that of sodium hydroxide was $1: 1.2$. The resultant mixture was transferred into a zirconia crucible and heated in a muffle furnace at a stable temperature of $593 \pm 1 \mathrm{~K}$ for $1.5 \mathrm{~h}$ at a heating rate of $275 \mathrm{~K}$ per minute. The fused material was allowed to cool and grinded with mortar and pestle. It was added to distilled water (solid to liquid ratio was 1: 10 $\mathrm{w} / \mathrm{w}$ ), and the slurry obtained was stirred in a glass beaker overnight. Microwave sintering was carried out by keeping under microwave irradiation at $100 \mathrm{~W}$ for the duration of $1 \mathrm{~h}$ to cure and crystallize the zeolitic composites. The final product was filtered and washed multiple times until the $\mathrm{pH}$ of the final wash was 10 . The resultant material was dried at $373 \pm 1 \mathrm{~K}$, cooled to room temperature and stored in a clean airtight container until needed.

Microwave alkaline hydrothermal method was used for the synthesis of MgMZBFA. A known mass of the mixture of the prepared BFA and sodium aluminate in the ratio of 1:0.5 was suspended into a predetermined volume of $1.0 \mathrm{M}$ sodium hydroxide solution (solid to liquid ratio being $1.0 \mathrm{~g}: 10 \mathrm{~mL}$ ) in a flat-bottom flask with constant stirring for $20 \mathrm{~min}$. The resulting suspension was irradiated with microwave energy of $900 \mathrm{~W}$, frequency $2.5 \mathrm{GHz}$ corresponding to a wavelength of $12.2 \mathrm{~cm}$ for $20 \mathrm{~min}$. The resultant solid was recovered by filtration and washed with distilled water for the removal of excess reactants. The solid labelled microwave zeolitic bagasse fly ash (MZBFA) was dried and kept in an airtight container until further use. MZBFA was treated with $1.0 \mathrm{M}$ magnesium chloride at room temperature while stirring for a period of $24 \mathrm{~h}$ for the incorporation of $\mathrm{Mg}^{2+}$ into the zeolite's frameworks. The solid-to-liquid ratio, in this case, is $1.0 \mathrm{~g}: 10 \mathrm{~mL}$. The resultant solid was filtered and washed with distilled water in order to remove excess $\mathrm{Mg}^{2+}$ on the surface. The excess removal was checked by adding few drops of Eriochrome Black T solution in basic medium. The solid was dried and kept in an airtight container. The $\mathrm{Mg}^{2+}$ incorporation was by external ion exchange method in order to avoid the precipitation of alkali earth metals in the alkaline aqueous medium which could hinder the zeolitization process.
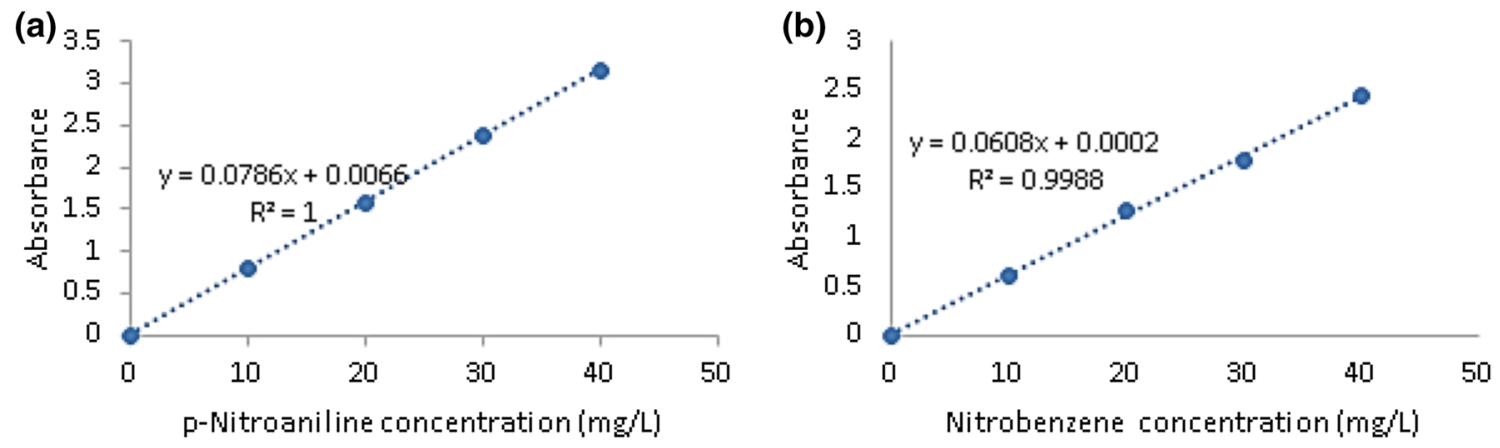

Fig. 1 Calibration plots for the measurement of a PNAN, b NB

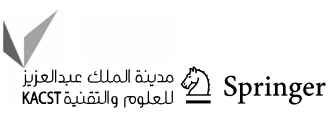


Table 1 Chemical compositions of BFA, and the synthesized adsorbents MgFZBFA and MgMZBFA

\begin{tabular}{llll}
\hline $\begin{array}{l}\text { Chemical constitu- } \\
\text { ents }(\%)\end{array}$ & BFA & MgFZBFA & MgMZBFA \\
\hline $\mathrm{SiO}_{2}$ & 83.14 & 53.16 & 67.64 \\
$\mathrm{Al}_{2} \mathrm{O}_{3}$ & 1.53 & 11.61 & 5.43 \\
$\mathrm{Na}_{2} \mathrm{O}$ & & 4.39 & 2.86 \\
$\mathrm{CaO}$ & 2.60 & 6.32 & 1.30 \\
$\mathrm{MgO}$ & 1.60 & 11.62 & 6.12 \\
$\mathrm{~K}_{2} \mathrm{O}$ & 4.23 & 1.06 & 5.21 \\
$\mathrm{Cl}$ & 0.05 & & \\
$\mathrm{Fe}_{2} \mathrm{O}_{3}$ & 6.06 & 7.39 & 6.96 \\
$\mathrm{MnO} \mathrm{O}$ & 0.22 & 0.23 \\
$\mathrm{TiO}_{2}$ & 0.22 & 0.96 & 0.68 \\
$\mathrm{SiO}_{2} / \mathrm{Al}_{2} \mathrm{O}_{3}$ & 0.29 & 4.57 & 12.45 \\
\hline
\end{tabular}

\section{Characterization of the sorbents}

\section{X-ray fluorescence (XRF)}

The BFA has very low alumina content compared to silica $\left(\mathrm{SiO}_{2} / \mathrm{Al}_{2} \mathrm{O}_{3}\right.$ by mass is 54.3) (Table 1$)$. The relative rise of alumina in MgFZBFA and MgMZBFA compared to BFA is due to aluminium supplementation by the inclusion of sodium aluminate in the syntheses. This was done to lower the $\mathrm{Si} / \mathrm{Al}$ ratio in order to boost the chance of zeolitization.

The alkali dissolution of the components of BFA precedes the formation of aluminosilicate gel which eventually crystallizes as zeolites. The $\mathrm{Si} / \mathrm{Al}$ ratio of MgFZBFA (obtained by alkali fusion synthesis method) is significantly lower than that of MgMZBFA (obtained by microwave alkaline hydrothermal synthesis method). This observation suggests that more dissolved silica was available for much $\mathrm{Al}$ incorporation during the formation of aluminosilicate gel in the case of the alkali fusion synthesis method than in the case of the alkaline hydrothermal method. This says much about the efficiency of the dissolution of the crystalline components of the BFA, most especially the silica components, and the chance of zeolitization. It could be inferred that the alkali fusion method (that produced MgFZBFA) is more efficient in terms of breaking down and solubilizing the silica constituents of BFA for zeolitization. Also, the better incorporation of $\mathrm{Al}$ in the fusion synthesis than in the hydrothermal synthesis suggests why there is greater incorporation of $\mathrm{Mg}(\mathrm{II})$ in MgFZBFA than in MgMZBFA. In zeolitization, $\mathrm{Al}^{3+}$ being triply charged and replacing some of $\mathrm{Si}^{4+}$ in their tetrahedral positions in the aluminosilicate structure results in the deficiency of positive charge which must be balanced in order to attain stability. Therefore, the greater incorporation of $\mathrm{Mg}(\mathrm{II})$ in $\mathrm{MgFZBFA}$ is because more Al had the chance to be introduced into the zeolitic frameworks. The proportion of zeolite phases in MgFZBFA is expected to be more than that of the alkaline hydrothermal product MgMZBFA.

\section{Fourier transform infrared spectroscopy (FTIR)}

FTIR suggests the zeolitic nature of the synthesized adsorbents. Table 2 characterizes the BFA and the synthesized zeolitic composite adsorbents: MgFZBFA; and MgMZBFA, by their IR absorption vibrations at specific wavenumbers. The IR vibrational spectra (Figs. 2, 3, and 4) show the changes that occur from the starting material BFA to the synthesized products MgFZBFA and MgMZBFA. The IR absorption bands $1150.85 \mathrm{~cm}^{-1}, 791.68 \mathrm{~cm}^{-1}$ and $473.53 \mathrm{~cm}^{-1}$ in BFA spectrum (Fig. 2) originate from the stretching and bending of the internal tetrahedral $\mathrm{TO}_{4}$ ( $\mathrm{T}=\mathrm{Si}, \mathrm{Al}$ ) corresponding to the asymmetrical stretching vibrations of $\mathrm{Si}-\mathrm{O}-\mathrm{Si}$; the symmetrical stretching vibrations of $\mathrm{Si}-\mathrm{O}-\mathrm{Si}$; and the bending vibrations of $\mathrm{O}-\mathrm{Si}-\mathrm{O}$ ,respectively (Scott et al. 2003; Tantawy et al. 2014). The broad band at $3395 \mathrm{~cm}^{-1}$ corresponds to the stretch band of - $\mathrm{OH}$ functional group and this can be associated with adsorbed water. For the synthesized products (Figs. 3 and 4), there is an increase in the intensities in the broad band associated with the stretching $-\mathrm{OH}$. This suggests

Table 2 FTIR characterization of BFA, MgFZBFA, and MgMZBFA showing different infrared absorption bands

\begin{tabular}{|c|c|c|c|c|}
\hline \multirow[t]{2}{*}{ Wavenumber $\left(\mathrm{cm}^{-1}\right)$} & \multirow[t]{2}{*}{ Possible assignments } & \multicolumn{3}{|c|}{ Observed bands $\left(\mathrm{cm}^{-1}\right)$} \\
\hline & & BFA & MgFZBFA & MgMZBFA \\
\hline $3800-3300$ & Stretching vibration of $-\mathrm{OH}$ group, silanol $(\mathrm{Si}-\mathrm{OH})$ & 3395 & 3440 & 3439 \\
\hline $1700-1400$ & $\mathrm{OH}$ deformation and bending vibration of interstitial water & & 1640 & 1638 \\
\hline $1250-850$ & $\begin{array}{l}\text { Asymmetric internal } \mathrm{T}-\mathrm{O} \text { stretching of } \mathrm{TO}_{4} \text { tetrahedra, } \mathrm{Si}-\mathrm{O}-\mathrm{Si} \\
(\mathrm{T}=\mathrm{Si}, \mathrm{Al})\end{array}$ & 1151 & 1018 & $1091 ; 1022$ \\
\hline $800-600$ & $\begin{array}{l}\text { Symmetric stretching of internal tetrahedra } \\
\mathrm{TO}_{4}\end{array}$ & 792 & 750 & 791 \\
\hline $420-500$ & $\begin{array}{l}\text { Bending mode of internal tetrahedra } \\
\mathrm{TO}_{4}(\mathrm{O}-\mathrm{T}-\mathrm{O})\end{array}$ & 473 & 460 & 473 \\
\hline
\end{tabular}




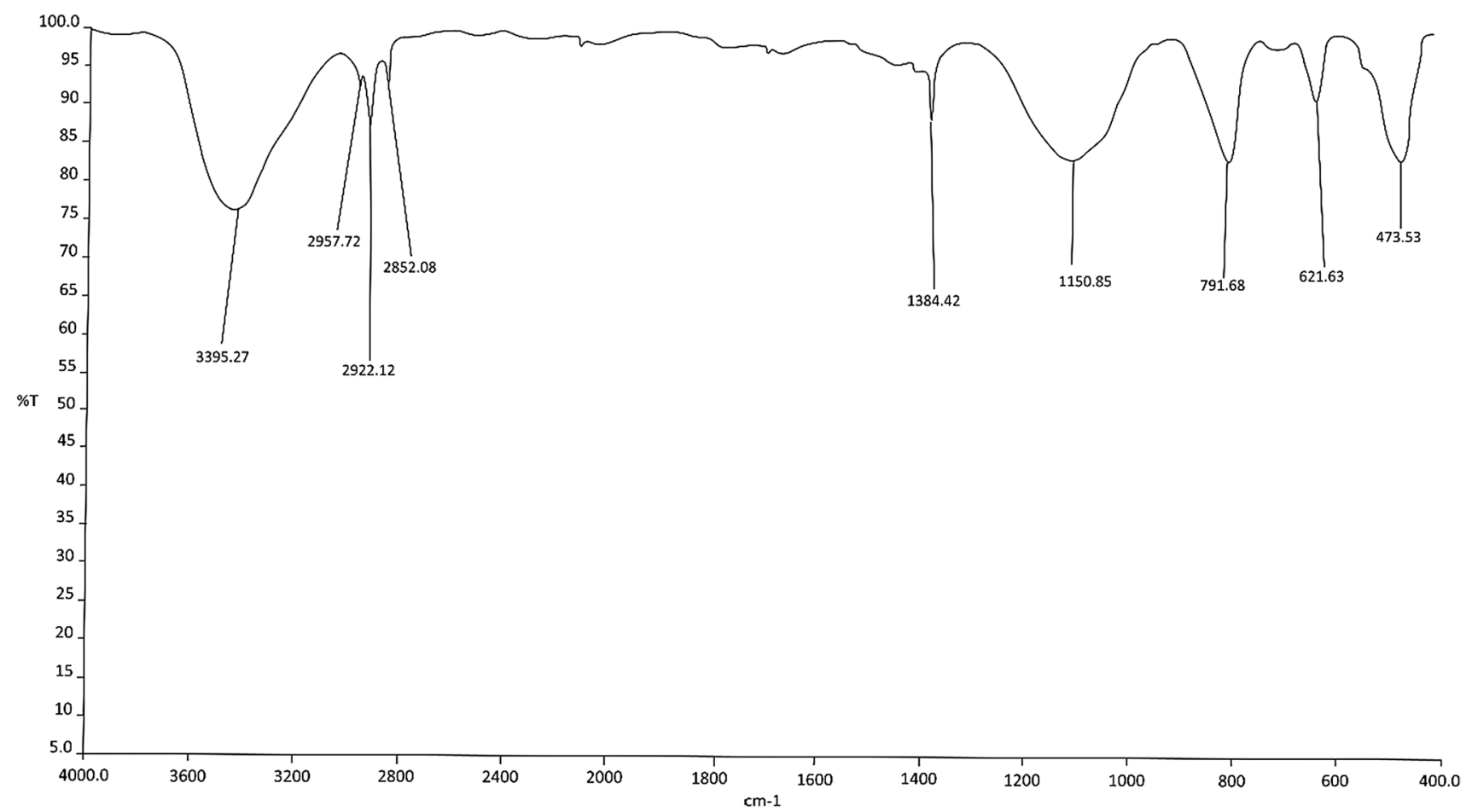

Fig. 2 FTIR absorption spectrum of BFA

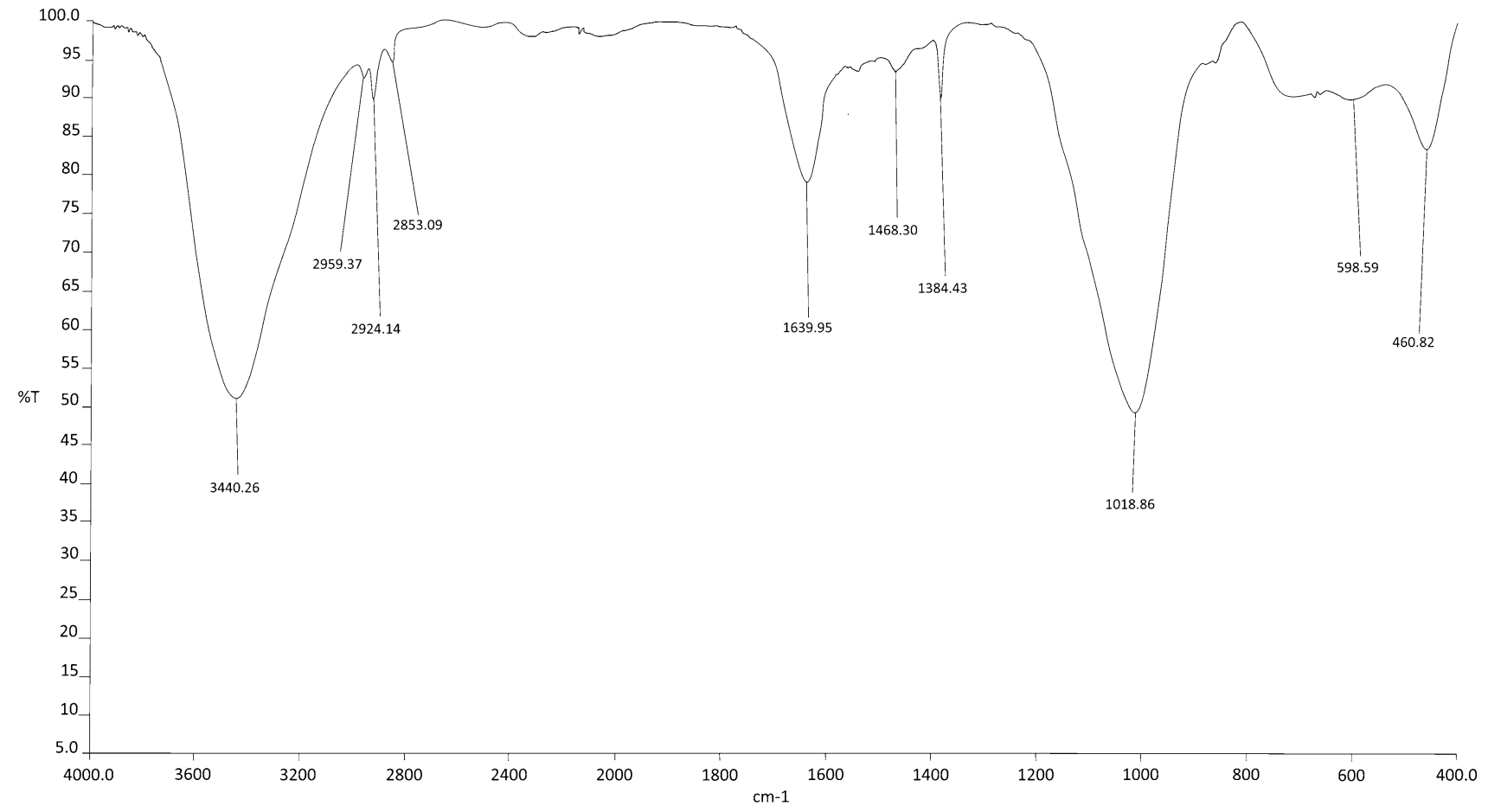

Fig. 3 FTIR absorption spectrum of MgFZBFA

the appearance of $\mathrm{Si}-\mathrm{OH}$ group which is a feature of a typical zeolite material (Shah et al. 2016). Also for the synthesized products, the bands that appear at $1637 \mathrm{~cm}^{-1}$ (which is noticeably absent in the spectrum of BFA) are vibrations peculiar to $-\mathrm{OH}$ functional group which can be ascribed to water of zeolitic nature (Hildebrando et al. 


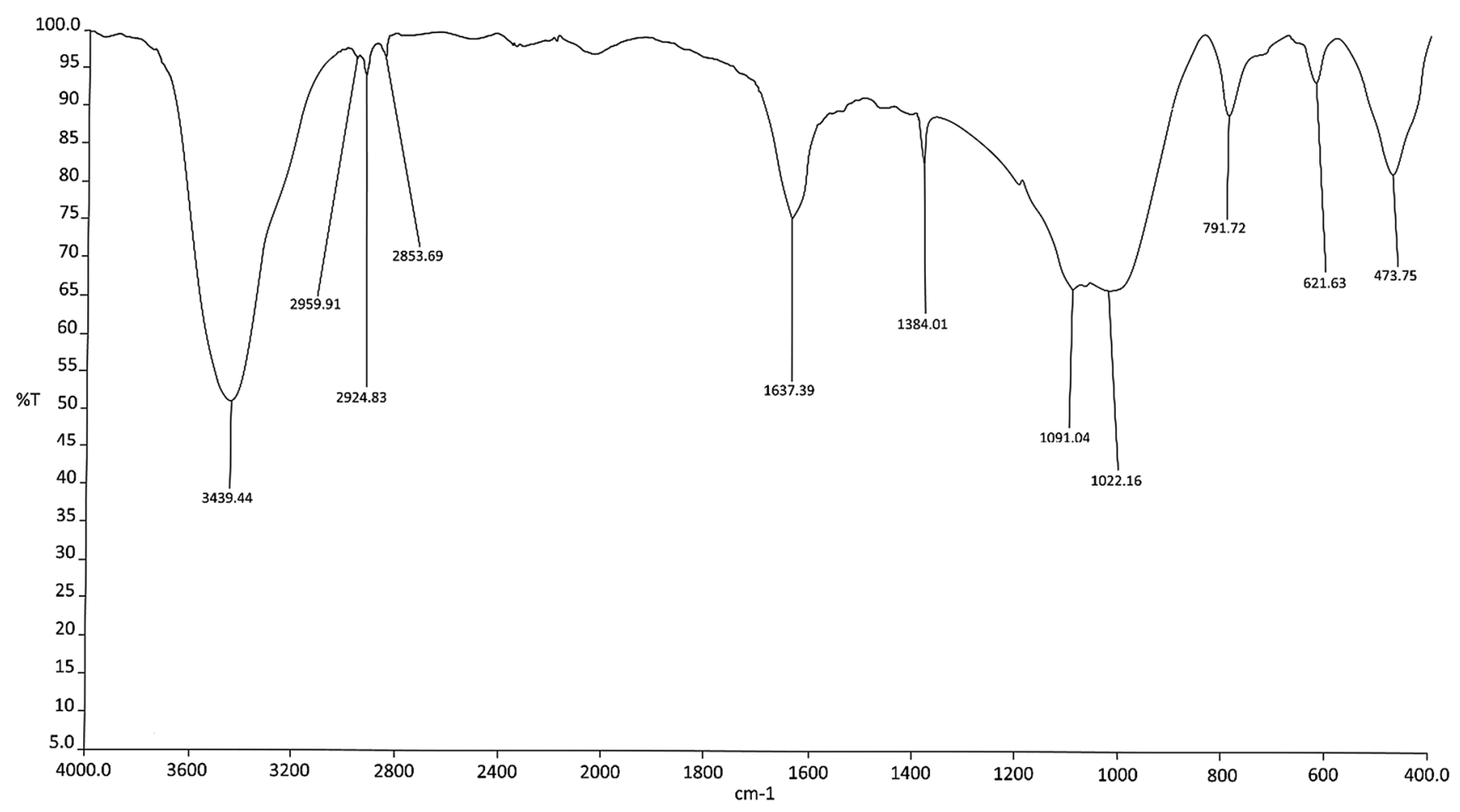

Fig. 4 FTIR absorption spectrum of MgMZBFA

2014). In addition, the $\mathrm{TO}_{4}$ bands in the synthesized products (initially at $1150 \mathrm{~cm}^{-1}$ in the spectrum of BFA) have shifted to lower wavenumbers and they appear to be more intense. It has been established that the wavenumber of this asymmetric stretching vibration band decreases with an increase in $\mathrm{Al}$ content. Hence, the shift of the $\mathrm{T}-\mathrm{O}$ band to lower wavenumber indicates the incorporation of $\mathrm{Al}$ in the $\mathrm{TO}_{4}$ of aluminosilicate to increase in the number of tetrahedrally positioned Al to form zeolites (Tanaka et al. 2002; Wu et al. 2008; Tantawy et al. 2014). The shift of the asymmetric $\mathrm{TO}_{4}$ bands to lower wavenumbers is conspicuously more in MgFZBFA than in MgMZBFA, and this is in absolute agreement with the result from XRF analysis which shows less Si/Al ratio for MgFZBFA than MgMZBFA. This further emphasizes the more efficient breaking down and dissolution of BFA components for

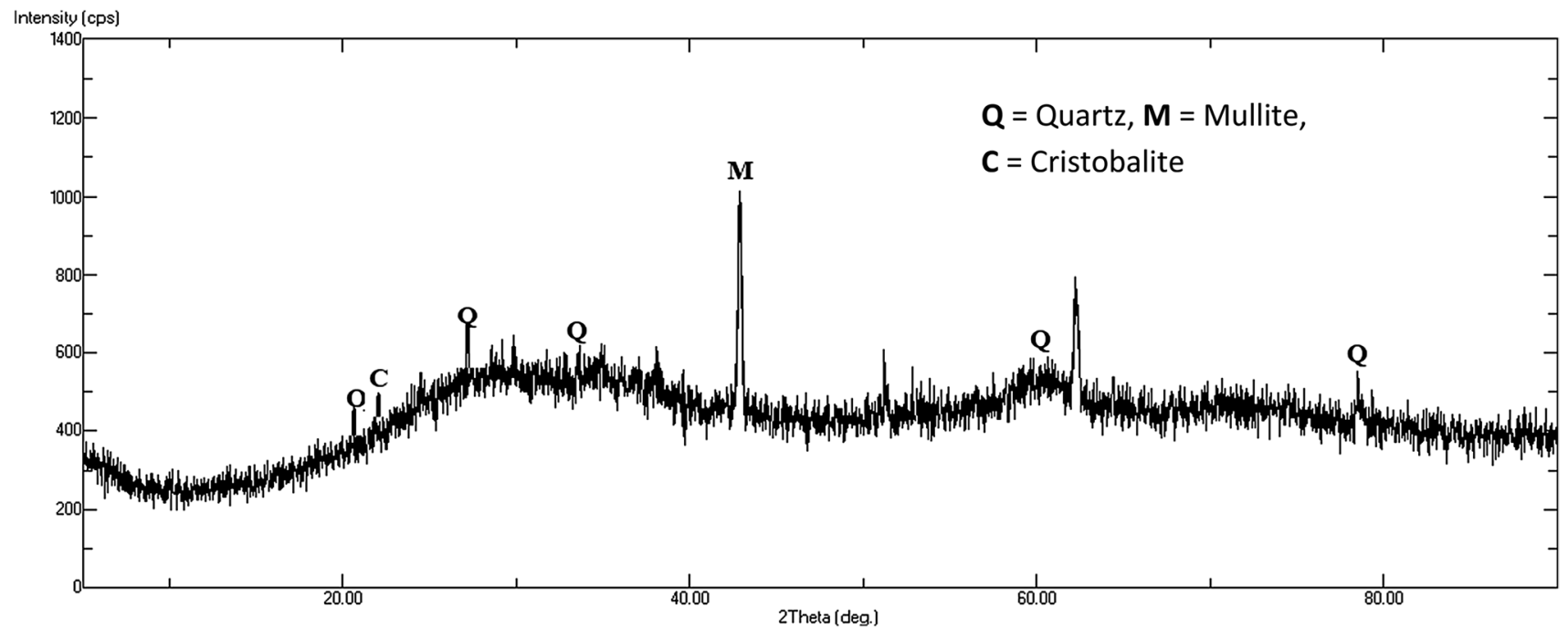

Fig. 5 PXRD spectrum of BFA showing constituent mineral phases 


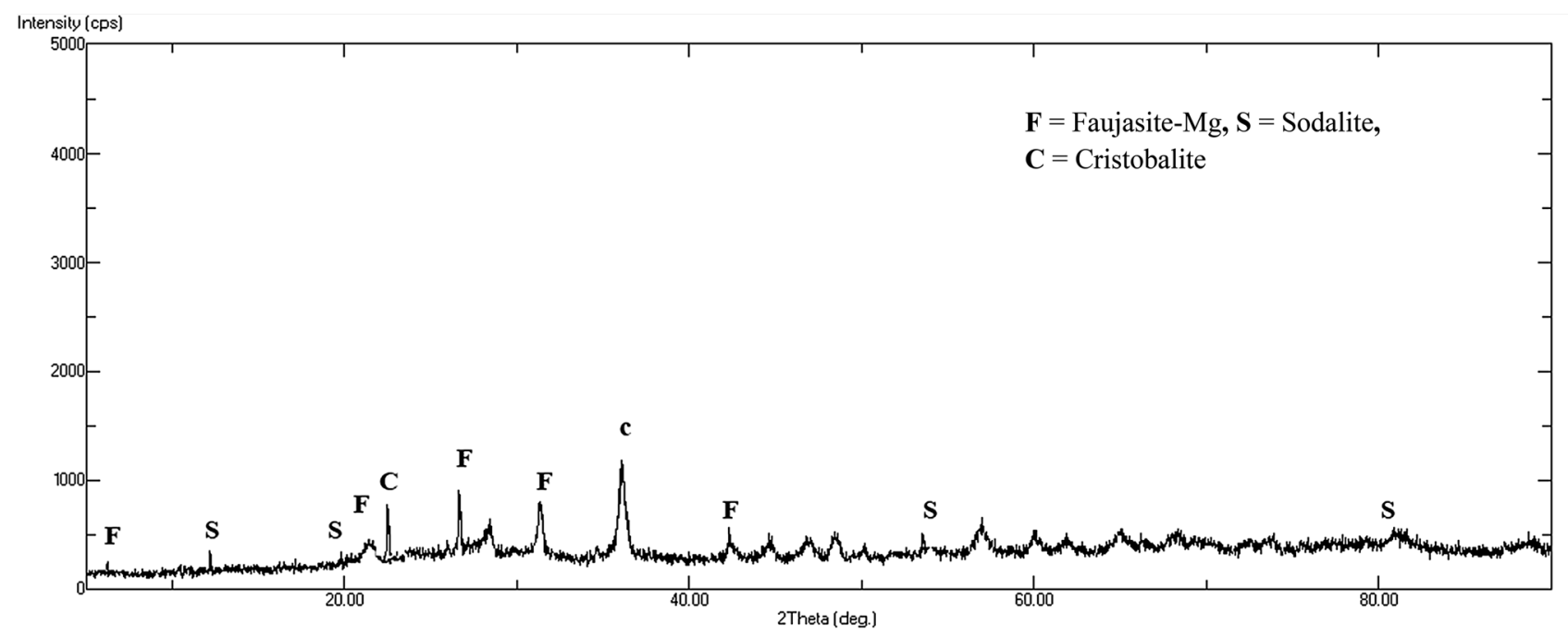

Fig. 6 PXRD spectrum of MgFZBFA showing constituent mineral phases

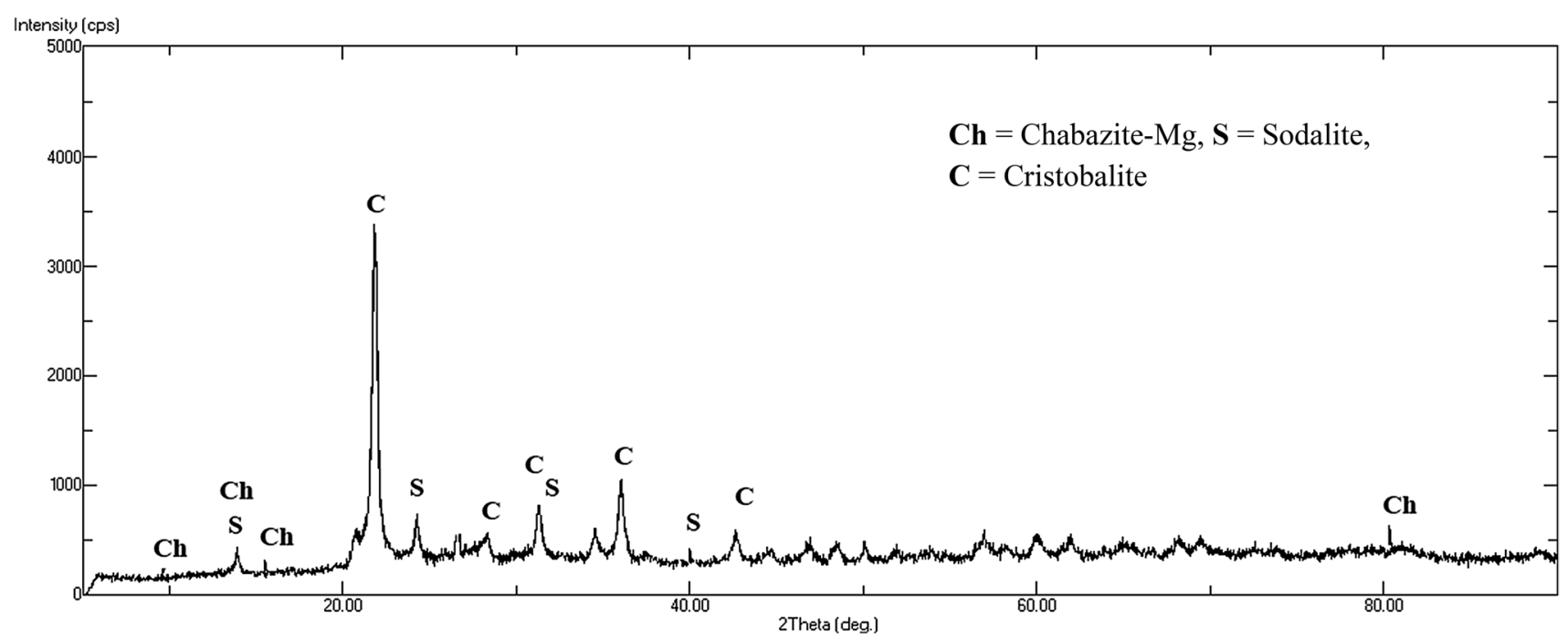

Fig. 7 PXRD spectrum of MgMZBFA showing constituent mineral phases

zeolitization in MgFZBFA than in MgMZBFA for the zeolitization process.

\section{Powder X-ray diffraction (PXRD)}

The PXRD spectra (Figs. 5, 6, and 7) also reflect some changes that occur from the starting material BFA to the syntheses MgFZBFA and MgMZBFA. The mineral phases represented by the diffractograms of BFA, MgFZBFA, and MgMZBFA were identified based on Crystallographic Open Database for Inorganic Compounds (COD-Inorg REV198327 2017.07.03). The synthesized samples were compared with the numerous reference patterns of inorganic phases present in the Crystallographic Open Database and by this, the mineral phases in the samples were identified.

The diffractogram for BFA (Fig. 5) shows a broad hump $\left(2 \theta=20-40^{\circ}\right)$ suggests that it contains a substantial amount of glassy amorphous phase. Silicon oxide diffractions such as that of quartz $\left(2 \theta=20.84^{\circ}, 26.64^{\circ}, 36.5^{\circ}, 60.08^{\circ}\right.$ and $\left.77.68^{\circ}\right)$ and cristobalite $\left(2 \theta=21.90^{\circ}\right)$ were, however, detected in the diffractogram. Diffraction attributed to mullite $\left(2 \theta=42.66^{\circ}\right)$ was also found.

Judging from the diffractograms of fusion method product MgFZBFA (Fig. 6), fusion method seems to be a very harsh method, as the initial constituents such as quartz, mullite and cristobalite seem almost completely broken down and can rarely be seen in the XRD of MgFZBFA. 
Furthermore, there is a massive disappearance of the BFA hump and the appearance of entirely new crystalline peaks can be observed. These observations suggest that the BFA had undergone some major transformations during the synthesis process which must have involved the major dissolution of the (amorphous and crystalline) silica and alumina phases that were present in the BFA into aluminosilicate gel that crystallized as zeolites. Diffraction peaks of zeolitic composites involving faujasite- $\mathrm{Mg}\left(2 \theta=6.06^{\circ}, 21.02^{\circ}\right.$, $26.64^{\circ}$ and $\left.31.22^{\circ}\right)$ and sodalite $\left(2 \theta=13.98^{\circ}, 19.78^{\circ}\right.$ and $82.44^{\circ}$ ) were detected in MgFZBFA.

For the microwave alkaline hydrothermal product MgMZBFA, massive disappearance of the BFA hump can also be observed (Fig. 7) but the diffraction peaks of silica, particularly those of cristobalites are still abundant in the diffractograms. This means the amorphous glassy phases were thoroughly digested while some crystalline phases, particularly cristobalite have not been digested completely. And they perchance become very obvious due to the dissolution and zeolitization of the more soluble amorphous glassy phases. The relative abundance of these $\mathrm{SiO}_{2}$ diffraction peaks in the two diffractograms suggests that the microwave alkaline hydrothermal method for the zeolitization of the fly ash was less efficient as regards the dissolution of some crystalline $\mathrm{SiO}_{2}$ phases than the fusion method. However, new and crystalline diffraction peaks were detected in the diffractogram of MgMZBFA, and this suggests some extent of transformations which have taken place. Diffraction peaks of chabazite- $\operatorname{Mg}\left(2 \theta=9.4^{\circ}\right.$, $13.98^{\circ}, 15.92^{\circ}$ and $\left.80.38^{\circ}\right)$ and sodalite $\left(13.8^{\circ}, 24.18^{\circ}\right.$, $31.40^{\circ}, 40.02^{\circ}$ and $59.90^{\circ}$ ) were discovered in the diffractogram of MgMZBFA.

\section{Scanning electron microscopy (SEM)}

The transformations of the surface morphology of BFA through the zeolitization process were observed under a scanning electron microscope. SEM images of BFA, MgFZBFA, and MgMZBFA were observed at $\times 2500$ magnification. The particles of the BFA appear to be spherical (Fig. 8a). The spherical appearance is as a result of cooling and solidification effect that the particles experience while being suspended in flue gases (Musyoka et al. 2013). In the SEM micrographs that represent the fusion synthesis product MgFZBFA (Fig. 8b), the particles appear to be in agglomerate form with rough morphologies, and the initial spherical shape of the BFA appears to be no more. This could be due to the chemical impact involved in the zeolitization process (Jha and Singh 2016), the impact brought about by the alkali treatment of BFA which resulted in the breaking down and solubilization of the mineral phases. The particles appear puffy which could be an indication of the development of pore volumes. In addition, MgFZBFA appears to be of greater surface area. For the microwave product MgMZBFA (Fig. 8c), there is also the development of rough, non-spherical and porous morphology.
Fig. 8 SEM micrographs of a BFA, b MgFZBFA, c MgMZBFA showing their morphologies
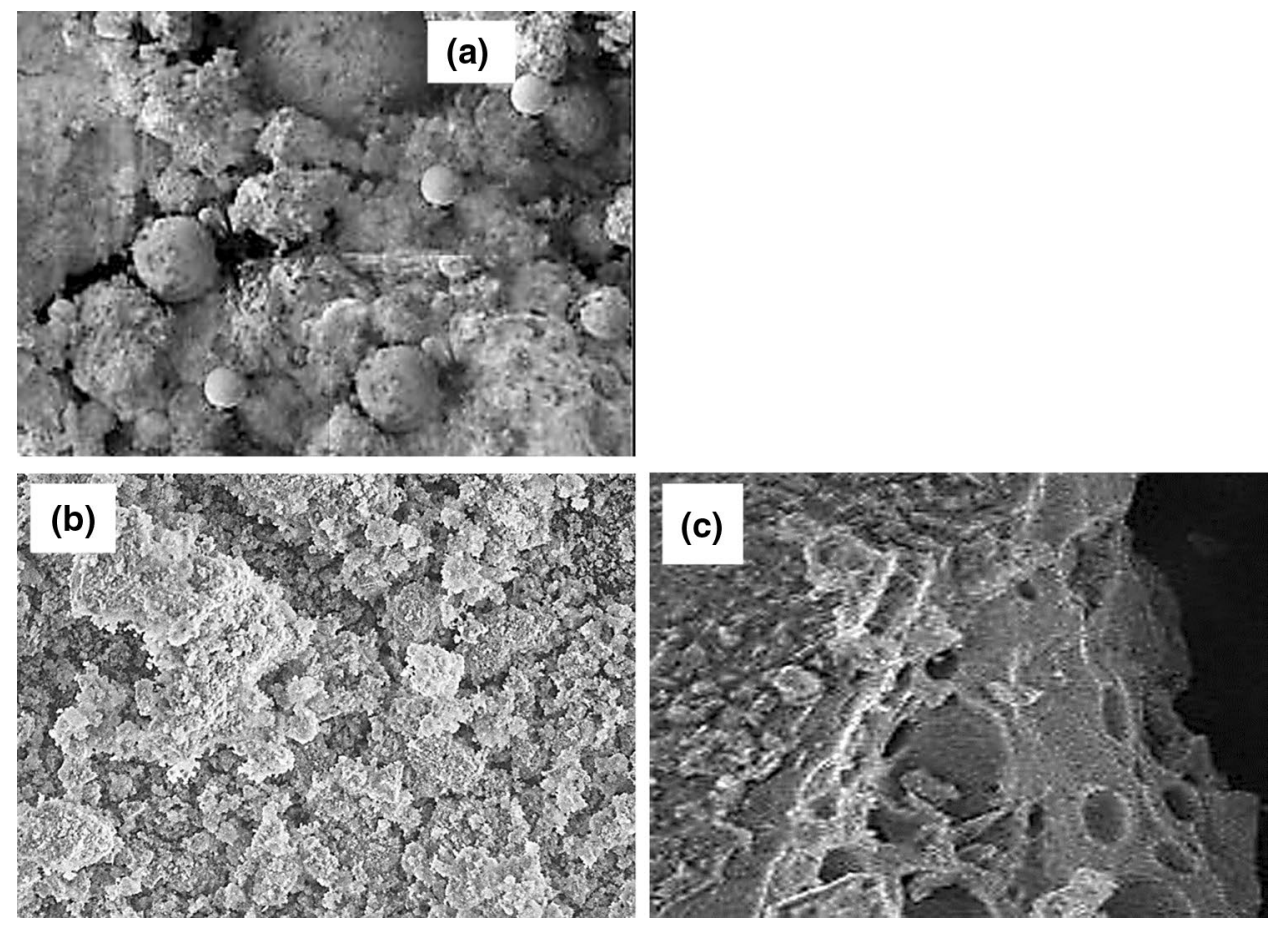
Table 3 Physicochemical properties of MgFZBFA and MgMZBFA

\begin{tabular}{|c|c|c|}
\hline Characteristics & MgFZBFA & MgMZBFA \\
\hline \multicolumn{3}{|l|}{ Proximate analysis } \\
\hline Moisture content (\%) & 12.70 & 2.02 \\
\hline Ash content $(\%)$ & 77.37 & 96.20 \\
\hline \multicolumn{3}{|l|}{ Physical properties } \\
\hline Bulk density & 0.33 & 0.69 \\
\hline $\mathrm{PZC}$ & 9.08 & 8.03 \\
\hline \multicolumn{3}{|l|}{ Instrumental methods } \\
\hline \multicolumn{3}{|l|}{ BET } \\
\hline Surface area $\left(\mathrm{m}^{2} \mathrm{~g}^{-1}\right)$ & 83.02 & 3.26 \\
\hline Adsorption average pore diameter (nm) & 13.82 & 17.18 \\
\hline Desorption average pore diameter (nm) & 13.82 & 17.18 \\
\hline \multicolumn{3}{|l|}{ BJH } \\
\hline $\begin{array}{l}\text { Adsorption cumulative pore volume } \\
\left(\mathrm{cm}^{3} \mathrm{~g}^{-1}\right)\end{array}$ & 0.29 & 0.01 \\
\hline $\begin{array}{l}\text { Desorption cumulative pore volume } \\
\left(\mathrm{cm}^{3} \mathrm{~g}^{-1}\right)\end{array}$ & 0.28 & 0.01 \\
\hline Desorption average pore diameter (nm) & 13.72 & 16.58 \\
\hline
\end{tabular}

\section{Surface area, pore volume, and bulk density}

Results obtained from the BJH/BET analysis of the adsorbent samples show that the surface area and the pore volume of MgFZBFA are greater than those of MgMZBFA (Table 3). The results obtained for the bulk densities of MgFZBFA and MgMZBFA are in accordance with the inverse trend of the cumulative pore volumes associated with the adsorbents, i.e., on comparing the two adsorbents, the decrease in the magnitude of density was observed with increase in the cumulative pores of the adsorbents. Hence, the bulk density of MgFZBFA is less than that of MgMZBFA. This analogy is consistent with the presumption that lower density of adsorbents could mean higher porosity due to the increase in the volume as a consequence of the development of more porous nature. Therefore, the density results are in concordance with the BET/BJH results that shows MgFZBFA to have better pore volume than MgMZBFA.

\section{Moisture and ash content}

Zeolites could often be hydrated at non-elevated temperatures, and water molecules and other volatiles could often be found on the surface and in the cavities. The adsorbed water content reflects the free volume in the zeolite cavities which could be accessible to adsorbates (Usachev et al. 2003; Majchrzak-Kuceba 2013). Therefore, the quantification of desorbed volatiles from an adsorbent of zeolitic nature could be used as a measure of porosity, especially when a comparison is involved. Thermogravimetric method was used to compare the sorbed moisture and the zeolitic water content of the synthesized adsorbents. When MgFZBFA and MgMZBFA were heated to about $473 \mathrm{~K}, \mathrm{MgFZBFA}$ gave a higher percentage of desorbed volatiles. This suggests a better porous property for MgFZBFA than MgMZBFA. And this is consistent with the results from BET/BJH which reveals that MgFZBFA possesses greater pore volume than MgMZBFA. In addition, the ash content or residue level of the two adsorbents were compared after subjecting them to thermal treatment to about $1273 \mathrm{~K}$. The residue level of MgFZBFA (77.37\%) was found less than that of MgMZBFA (96.20\%). This suggests that for a unit gram of the synthesized adsorbents, some components such as zeolitic waters (which are the water molecules from the channels/ void system of the zeolites structures, and water from the dehydration of hydrated cations positioned in the zeolites frameworks) and some of the adsorbable volatiles including sorbed water that might have been driven off by thermal energy may be more abundant in MgFZBFA. Hence, it could be suggested that MgFZBFA possess more porous zeolitic characters than MgMZBFA.

\section{Point of zero charge (PZC)}

The PZC is a very important property of an adsorbent that enhances the understanding of the possible mechanisms responsible for the adsorbent-adsorbate interactions that cause sorption. If the PZC of an adsorbent is known, the net surface charge of the adsorbent at a particular $\mathrm{pH}$ and its influence on the sorption of a target adsorbate can be predicted. Hence, the PZC is always taken into consideration. The $\mathrm{pH}$ drift method was used to determine the PZC of the MgFZBFA and MgMZBFA. $75 \mathrm{mg}$ weights of an adsorbent were equilibrated with $25 \mathrm{~mL}$ of $0.01 \mathrm{~N} \mathrm{NaCl}$ solutions of different $\mathrm{pH}$ values $2 ; 4 ; 6 ; 8 ; 10$. The eventual $\mathrm{pH}$ of each suspension was obtained and used for the estimation of the PZC of each adsorbent (Fiol and Villaescusa 2009; Mahmood et al. 2011; Cardenas-Pena et al. 2012; Tran et al. 2017). The PZC values of the adsorbents were obtained from their PZC plots (Fig. 9). They are the plots of change in $\mathrm{pH}$ against the initial $\mathrm{pH}$ (values obtained from the $\mathrm{pH}$ drift procedure for estimating PZC). The equations of the straight lines are given in the plots. And the PZC values of the adsorbents were obtained from the horizontal intercepts of these plots, that is, the initial $\mathrm{pH}$ values at which the change in $\mathrm{pH}$ is null. The PZC values obtained for, MgFZBFA, and MgMZBFA are 9.08 and 8.03, respectively.

\section{Adsorption studies}

The goal of an adsorption experiment is to determine the adsorption performance or adsorption capacity of an adsorbent for a known adsorbate, at known experimental conditions such as $\mathrm{pH}$, concentration, dosage, etc. At each

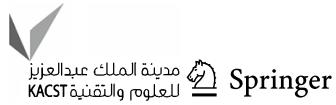



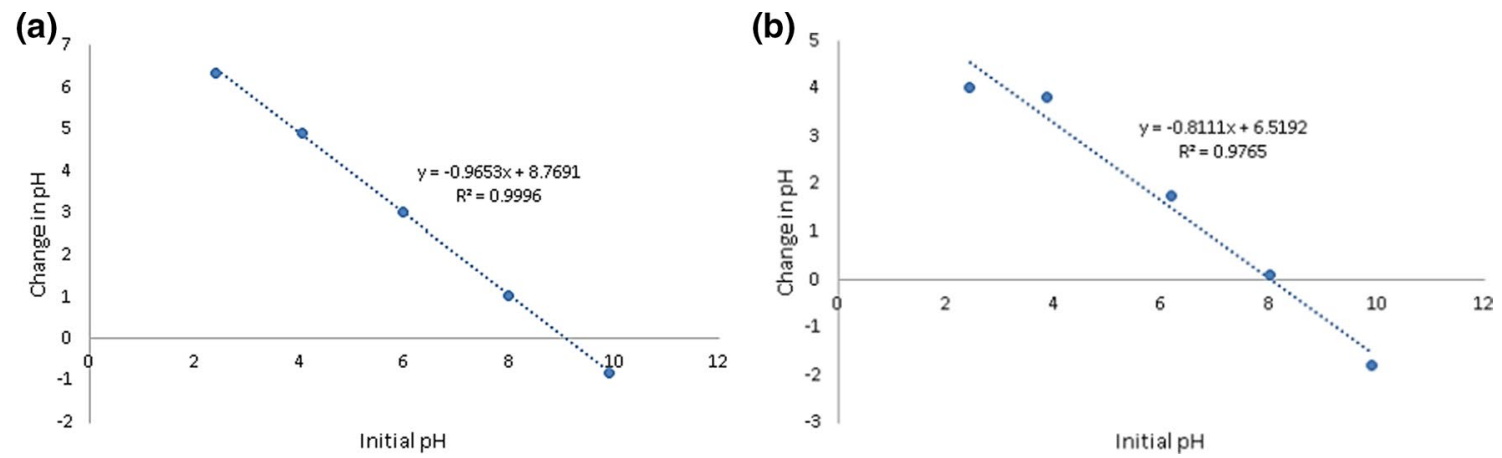

Fig. 9 PZC plots for a MgFZBFA, b MgMZBFA

experimental condition, the adsorption performances could be expressed as a measure of the percentage of the adsorptive removed from the solution by adsorption which is mathematically expressed as:

$\% E=\frac{\left(C_{\mathrm{o}}-C_{\mathrm{e}}\right)}{C_{\mathrm{o}}} \times 100$,

or as a measure of the amount of the adsorptive sorbed by a unit mass of adsorbent ' $q$ ' which is mathematically expressed as:

$q_{\mathrm{e}}(\mathrm{mg} / \mathrm{g})=\frac{\left(C_{\mathrm{o}}-C_{\mathrm{e}}\right)}{m}$,

where $C_{\mathrm{o}}$ in $\mathrm{mg} \mathrm{L}^{-1}$ is the initial concentration of adsorptive, $C_{\mathrm{e}}$ in $\mathrm{mg} \mathrm{L}^{-1}$ is the concentration of adsorptive at adsorption equilibrium, and $m$ is the adsorbent dose in $\mathrm{g} \mathrm{L}^{-1}$.

In this work, adsorption experiments were carried out at several $\mathrm{pH}$ values $(\mathrm{pH} 3-10)$ of the aqueous solutions of the pollutants adjusted with drops of $0.5 \mathrm{M}$ hydrochloric acid and $0.5 \mathrm{M}$ sodium hydroxide; with several doses of the adsorbents $\left(0.25-5 \mathrm{~g} \mathrm{~L}^{-1}\right)$, and with several concentrations of PNAN and NB (25-200 $\left.\mathrm{mg} \mathrm{L}^{-1}\right)$. Equilibrations of the adsorbents with the pollutants' solutions at the various experimental conditions and at $298 \mathrm{~K}$ were carried out with an orbital shaker at stirring speed of $150 \mathrm{rpm}$. Each adsorption treatment was carried out at different durations and adsorption measurements were taken at the end of each duration. Equilibrium was then said to be reached at the point when the concentration of the adsorptive in solution remained constant. The adsorption treatments were centrifuged and the filtrates were analysed for the residual or equilibrium concentration of the adsorbate $\left(C_{\mathrm{e}}\right)$, which can be used for the estimation of adsorption performance.

\section{Adsorption isotherms}

Adsorption isotherms describe the relationship established between the amount of the adsorbate sorbed by an adsorbent and the concentration of adsorptive left in solution during equilibrium at constant temperature (Dias et al. 2015). Suitable isotherm provides a description of an adsorption process. Two adsorption isotherm models: Langmuir and Freundlich, were examined in order to predict the mechanism for the adsorption processes.

The Langmuir model is based on monolayer adsorption on the active sites of the adsorbents (Amodu et al. 2015). The linear form of the equation is given as:

$\frac{C_{\mathrm{e}}}{q_{\mathrm{e}}}=\frac{1}{Q_{\mathrm{L}} K_{\mathrm{L}}}+\frac{C_{\mathrm{e}}}{Q_{\mathrm{L}}}$

$q_{e}\left(\mathrm{mg} \mathrm{g}^{-1}\right)$ is the amount of the adsorptive removed (per a unit weight of the adsorbent) at equilibrium, $C_{\mathrm{e}}\left(\mathrm{mg} \mathrm{L}^{-1}\right)$ is the concentration of the adsorptive left (in solution) at equilibrium, $K_{\mathrm{L}}\left(\mathrm{L} \mathrm{mg}^{-1}\right)$ is Langmuir constant and $Q_{\mathrm{L}}\left(\mathrm{mg} \mathrm{g}^{-1}\right)$, which can be obtained from the linear plot of $\frac{C_{\mathrm{e}}}{q_{\mathrm{e}}}$ against $C_{\mathrm{e}}$, is the monolayer maximum adsorption capacity of the adsorbent when its surface is saturated with the adsorbate molecules.

A dimensionless constant, known as separation factor $R_{\mathrm{L}}$ evaluates an adsorption process in terms of favorability of occurrence, linearity and reversibility. $\left(0<R_{\mathrm{L}}<1\right)$ suggests that the adsorption is favorable, $\left(R_{\mathrm{L}}>1\right)$ suggests that the adsorption is unfavorable, $\left(R_{\mathrm{L}}=1\right)$ suggests that the adsorption is linear, and $\left(R_{\mathrm{L}}=0\right)$ suggests that the adsorption is irreversible (Dias et al. 2015). The separation factor is mathematically defined as:

$R_{\mathrm{L}}=\frac{1}{1+K_{\mathrm{L}} C_{\mathrm{o}}}$,

where $C_{\mathrm{o}}\left(\mathrm{mg} \mathrm{L}^{-1}\right)$ is the initial concentration of the adsorptive, and $K_{\mathrm{L}}$ is the Langmuir constant.

Freundlich isotherm model assumes multilayer adsorption (Kalavathy et al. 2005). The linear form is expressed as:

$\log q_{\mathrm{e}}=\log K_{\mathrm{F}}+\frac{1}{n} \log C_{\mathrm{e}}$ 
$K_{\mathrm{F}}$ is an indicator of the adsorption capacity which increases with the increase in the maximum adsorption capacity. $\frac{1}{n}$ also is a measure of the intensity of adsorption. The values of $K_{\mathrm{F}}$ and $\mathrm{n}$ can be obtained from the linear graph of $\log q_{\mathrm{e}}$ against $\log C_{\mathrm{e}}$. The adsorption becomes more favorable with an increase in the intensity of the adsorption. Generally, $n<1$, $\frac{1}{n}>1$ (UW Adsorption Equilibria Principles 2018).

\section{Sorption dynamics}

The uptakes of the pollutants from their aqueous solutions at time $t$ of equilibration with the adsorbents were quantified using the equation:

$q_{\mathrm{t}}=\frac{C_{\mathrm{o}}-C_{\mathrm{t}}}{m}$,

where $C_{\mathrm{o}}$ (whose unit is $\mathrm{mg} \mathrm{L}^{-1}$ ) is the concentration of the pollutant prior to sorption, $C_{\mathrm{t}}$ (unit in $\mathrm{mg} \mathrm{L}^{-1}$ ) is the concentration of the remaining pollutant after sorption for the time $t$, (in min) and $m$ (whose unit is $\mathrm{g} \mathrm{L}^{-1}$ ) is the adsorbent dose.

And the experimental data obtained were applied for the evaluation of kinetic models that describe the sorption processes. Pseudo-first-order kinetic model, pseudo-second-order kinetic model, and Weber and Morris diffusion model are considered in this work.

The pseudo-first-order kinetics model is of the assumption that the concentration of the pollutant adsorbate in the bulk aqueous solution is the rate-determining factor. The linear equation for this model is expressed as:

$\log \left(q_{\mathrm{e}}-q_{\mathrm{t}}\right)=\log q_{\mathrm{e}}-\frac{k_{1}}{2.303} t$,

where $q_{\mathrm{e}}$ and $q_{\mathrm{t}}$ (whose units are both $\mathrm{mg} \mathrm{g}^{-1}$ ) are the amount of adsorbed sorbate at equilibrium and at time $t$, respectively. $k_{1}$ (whose unit is $\min ^{-1}$ ) is the rate constant of the adsorption process that follows this model. The values of $k_{1}$ and $q_{\mathrm{e}}$ can be obtained from the linear graph of $\log \left(q_{\mathrm{e}}-q_{\mathrm{t}}\right)$ against $t$.

The pseudo-second-order adsorption kinetics model is of the assumption that the rate-determining step in an adsorption process is the sorption interaction on the surface of the adsorbent, regardless of adsorbate concentration in bulk solution (Liu 2008). The rate equation for this kinetic model is expressed as:

$\frac{t}{q_{\mathrm{t}}}=\frac{1}{k_{2} q_{\mathrm{e}}^{2}}+\frac{t}{q_{\mathrm{e}}}$

where $k_{2}$ (whose unit is $\mathrm{g} \mathrm{mg}^{-1} \mathrm{~min}^{-1}$ ) is the rate constant. $k_{2}$ and $q_{\mathrm{e}}$ can be obtained from the linear plots of $\frac{t}{q_{\mathrm{t}}}$ against $t$. Another parameter, the initial adsorption rate $h_{\mathrm{o}}$ (whose unit is $\mathrm{mg} \mathrm{g}^{-1} \mathrm{~min}^{-1}$ ) is expressed as: $h_{\mathrm{o}}=k_{2} q_{\mathrm{e}}^{2}$

The Weber and Morris intra-particle diffusion model was used to complementing the Langmuir adsorption isotherm model, Freundlich adsorption isotherm model, pseudofirst-order kinetics model and pseudo-second-order kinetics model, in order to further characterize the sorption processes and predict more the adsorption mechanisms. Same data from the time study experiments were examined with this model.

The intra-particle diffusion equation (Weber Jr and Morris 1963) is expressed as:

$q_{\mathrm{t}}=k_{\mathrm{i}} t / 2+I$,

where $q_{\mathrm{t}}$ (whose unit is $\mathrm{mg} \mathrm{g}^{-1}$ ) is the amount of the pollutant adsorbate sorbed by a unit mass of the adsorbent at a known time, $k_{\mathrm{i}}$ (whose unit is $\mathrm{mg} \mathrm{g}^{-1} \mathrm{~min}^{-1 / 2}$ ) is the intraparticle diffusion rate constant. $I$ (whose unit is $\mathrm{mg} \mathrm{g}^{-1}$ ) is the intercept which indicates the extent of the boundary layer effect. The larger its value, the more immense the contribution of the surface adsorption as the rate-determining step in an adsorption process (Sarkar et al. 2003). The mechanisms of the adsorption process $\$$ s were inspected using the straight-line plots of $q_{\mathrm{t}}$ against $t / 2$.

\section{Regeneration and reusability studies}

Regeneration studies for MgFZBFA and MgMZBFA were carried out in order to determine their reutilization potential. This was done using solvents to desorb the sorbed pollutants and render the adsorbents available for subsequent sorption rounds. For the desorption of PNAN, the adsorbents loaded with the pollutant (at the optimal sorption conditions) were shaken with $0.2 \mathrm{M}$ hydrochloric acid. A similar method was used for the desorption of NB but with $0.2 \mathrm{M}$ acetic acid. The recovered adsorbents were washed with distilled water and dried. They were again loaded with the pollutants' adsorbates for another adsorption-desorption cycle.

\section{Results and discussion}

\section{Effect of solution hydronium ion concentration}

For the $\mathrm{pH}$ study, several $25 \mathrm{~mL}$ of a predetermined concentration of a pollutant solution at different $\mathrm{pH}$ values (3-10) were equilibrated with a predetermined mass of an adsorbent in 100 -mL Erlenmeyer flasks.

Aside the interplay of the dispersion interactions such as van der Waals forces and hydrogen bonding (possibly from the interaction between the silanol group of zeolite phases and the amine and nitro groups of PNAN), $\mathrm{pH}$ was 
Fig. 10 Scheme of adsorption involving PNAN and the sorbents in a electro-attractive, b electro-repulsive conditions

\section{Resonance Hybrid for p-Nitro Aniline}

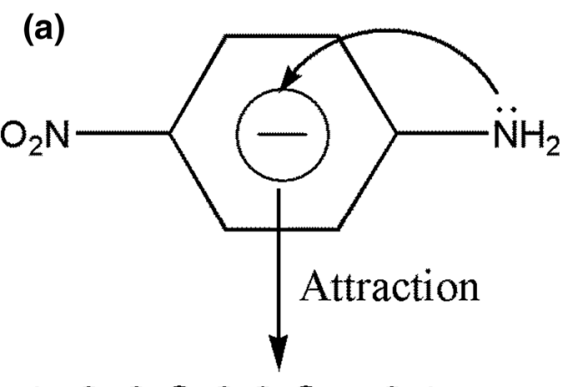

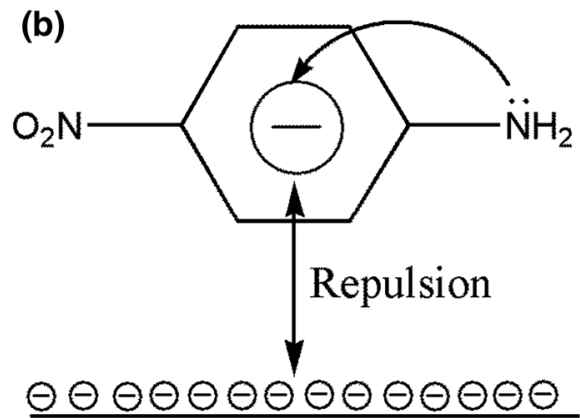

(b)

$\mathrm{pH}>\mathrm{PZV}$

\section{Adsorbent
Surface}

observed to have an effect on the adsorption of PNAN. At pH 3-7 (where the net surface charges of MgFZBFA and MgMZBFA are positive), PNAN (having the tendency to possess negative sites which is induced by the mesomeric effect caused by the interaction of the lone pair of electrons on the amine functional group with the aromatic ring) could undergo attractive electrostatic interactions with the positive surfaces of the adsorbents (Fig. 10).

However, the adsorption performances seem to drop at higher $\mathrm{pH}$ values (8-10) where the net surface charge of the adsorbents advance in the negative direction (Fig. 11a). Hence, $\mathrm{pH} 4$ which is significantly less than the PZC of the two adsorbents was selected as the optimal $\mathrm{pH}$ for the adsorption processes. At this $\mathrm{pH}$, the amounts of PNAN sorbed by MgFZBFA and MgMZBFA are $21.0 \mathrm{mg} \mathrm{g}^{-1}$ and $9.5 \mathrm{mg} \mathrm{g}^{-1}$, respectively.

NB is non-ionizable by a proton or hydroxyl ion. Hence, the variation in the adsorption performances depended solely on the surface charges of the adsorbents as the $\mathrm{pH}$ of the solutions are varied. The adsorption performances increased with increase in $\mathrm{pH}$ and the optimal adsorptions were obtained at $\mathrm{pH}$ values close to the PZC of the adsorbents (Fig. 11b). The behaviour can be explained as, the nitro group on NB being a deactivating group rendered the aromatic ring electron deficient, hence the acquisition of positive charge. The positive charge has the tendency to interact repulsively with the positive surfaces of the adsorbents (at $\mathrm{pH}<\mathrm{PZC}$ ). However, the gradual rise in the adsorption performances as $\mathrm{pH}$ increases could be explained by the reduction in the repulsive electrostatic interactions between NB and the adsorbents as the positive charge densities of the adsorbents dwindle when the $\mathrm{pH}$ values tend to approach PZC. The amounts of NB sorbed by MgFZBFA and MgMZBFA are $19.2 \mathrm{mg} \mathrm{g}^{-1}$ and $8.1 \mathrm{mg} \mathrm{g}^{-1}$, respectively, at the optimal $\mathrm{pH}(\mathrm{pH}$ 9).

At the optimal $\mathrm{pH}$ for each of the adsorption processes, it can be deduced from the adsorption plots that PNAN was better sequestrated by each of the adsorbents MgFZBFA and MgMZBFA because its sorption produced the higher $q_{\mathrm{e}}$ values compared to NB. The possible reason for this could
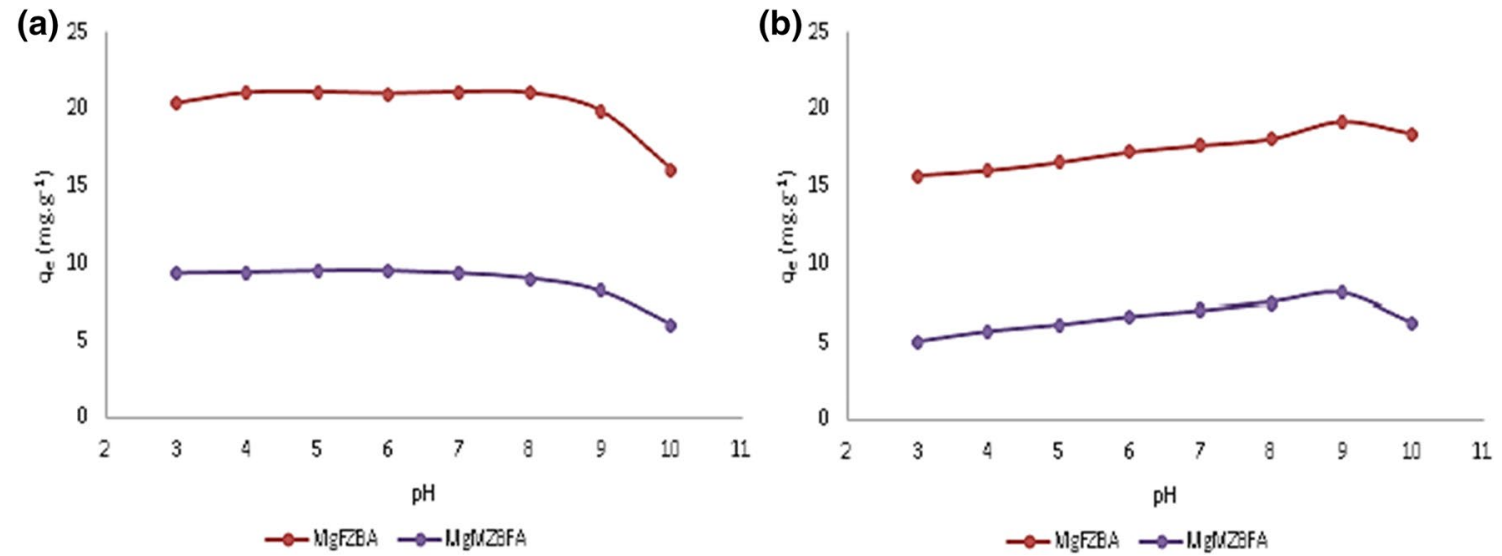

Fig. 11 Effect of $\mathrm{pH}$ on the sorption of a PNAN, b NB on MgFZBFA and MgMZBFA 
be the difference in the electronegativity/electropositivity properties of the pollutants which give them the potential to electrostatically interact with the surfaces of the adsorbents. The electronegative property of PNAN is similar to that of aniline and obtained by the partial delocalization of the lone pair of an electron on its nitrogen into the pi system of the benzene ring. On the other hand, NB has less electronegative or more of electropositive property due to the electron pulling effect of the nitro group from the pi system of the aromatic ring. It could be expected that the positive charge developed on the aromatic ring as electron density diminishes should bring about attractive electrostatic interaction with the negative surfaces of the adsorbents (especially at $\mathrm{pH}$ values beyond the PZC of the adsorbents) and probably cause significant sorption performance, but the sorption effect of these envisaged attractive electrostatic interactions seems insignificant.

\section{Effect of adsorbents dose}

The adsorbents dose experiments were performed at the optimum $\mathrm{pH}$ for each of the adsorption systems with predetermined concentrations of the pollutants PNAN and NB. For the experiments, doses of the adsorbents MgFZBFA and MgMZBFA were varied from $0.25-5.0 \mathrm{~g} \mathrm{~L}^{-1}$. Results (Fig. 12) show that the percentage adsorption of PNAN and NB increase with the adsorbents dose. However, there is obvious wane in the rate of the pollutants adsorption beyond $2.0 \mathrm{~g} \mathrm{~L}^{-1}$ doses of the adsorbents. The rise in the pollutants uptake with increasing dosage could be attributed to the increased number of adsorption sites as the adsorbents dose increase. The decline in the adsorption rate could be explained by possible aggregation effect of the adsorbents as the dose increases. This effect might have relatively diminished the extent to which adsorption sites were rendered available for the pollutants in the aqueous solutions. The adsorbents dose of $2.0 \mathrm{~g} \mathrm{~L}^{-1}$ was selected as the optimal dose for the adsorption systems in order to lessen the effect of the aggregation, get the best out of adsorbents-adsorbates interactions and augment effective adsorbates uptake.

\section{Effect of initial concentration}

The effect of the initial concentration of the pollutants in aqueous solutions was determined at the optimal $\mathrm{pH}$ and dose for the adsorption systems. In the adsorption processes involving the adsorbents, MgFZBFA and MgMZBFA, and the pollutants, PNAN and NB, an increase in the concentration of the pollutants in aqueous solutions caused an increase in the amount of the pollutants adsorbed per unit masses of the adsorbents $\left(q_{\mathrm{e}}\right)$ (Fig. 13). However, at some points of the concentration increase, there seems to be no significant increase in $q_{\mathrm{e}}$. In the adsorption curves, $100 \mathrm{mg} \mathrm{L}^{-1}$ seems to be the limit of $q_{\mathrm{e}}$ increase with the increase in the concentrations of the pollutants. Hence $100 \mathrm{mg} \mathrm{L}^{-1}$ was chosen as the optimum concentration for the adsorption systems.

\section{Effect of temperature}

The adsorption performances were evaluated at different temperatures. Results show that the amount of PNAN sorbed $\left(q_{\mathrm{e}}\right)$ in PNAN-adsorbents systems increase with the rise in temperature. At the temperatures of at $298 \mathrm{~K}, 308 \mathrm{~K}$, $318 \mathrm{~K}$ and $328 \mathrm{~K}$, the amounts of PNAN sorbed by MgFZBFA are 20.9, 21.6, 22.8, and $23.5 \mathrm{mg} \mathrm{g}^{-1}$, respectively, and the amounts of PNAN sorbed by MgMZBFA are 9.5, 10.1, 10.9 , and $11.5 \mathrm{mg} \mathrm{g}^{-1}$, respectively. This could be due to the increase in the possibility of PNAN interactions with the adsorbents. The increase in temperature could enhance the
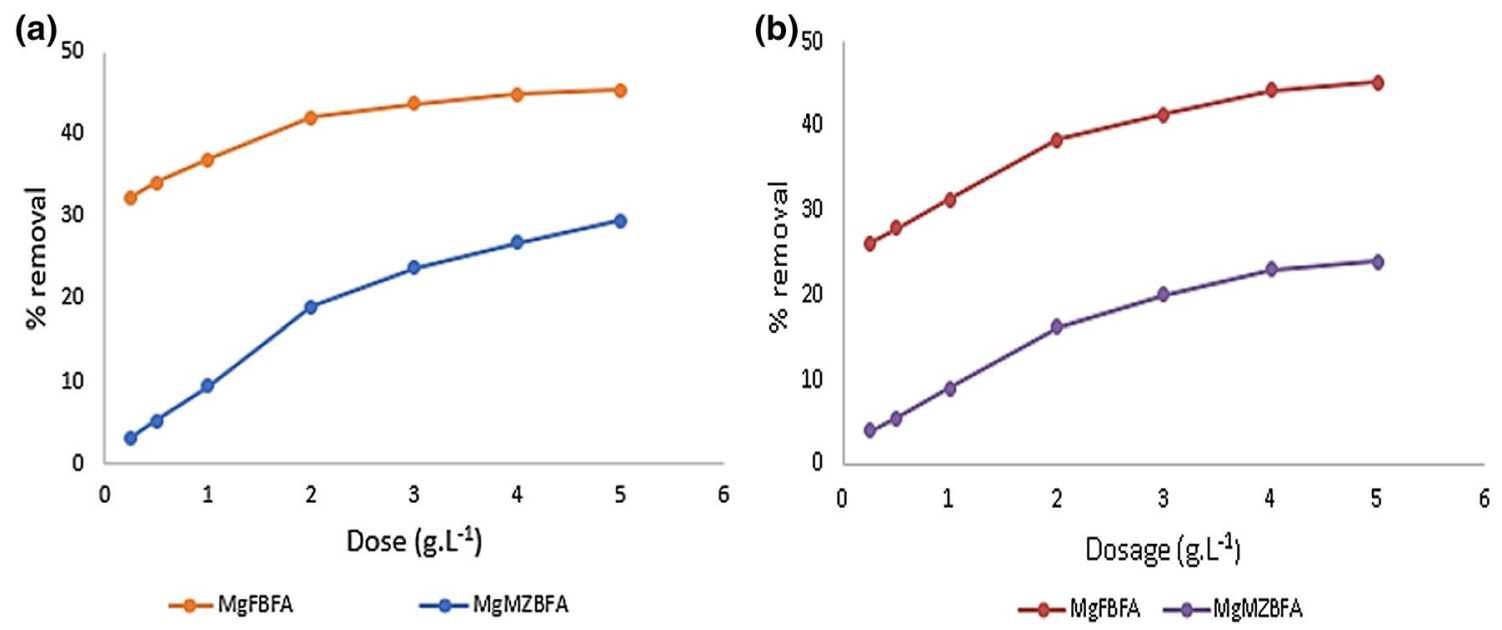

Fig. 12 Effect of adsorbents dose on the sorption of a PNAN, b NB on MgFZBFA and MgMZBFA 

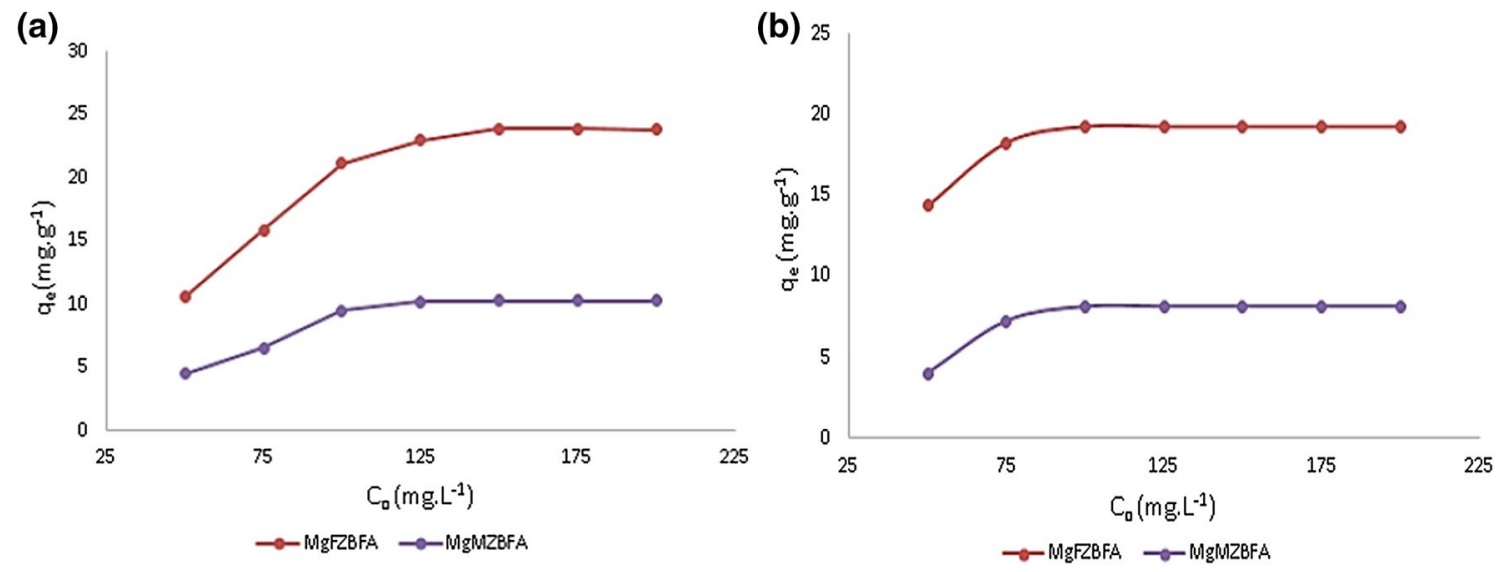

Fig. 13 Effect of initial concentration on the sorption of a PNAN, b NB on MgFZBFA and MgMZBFA

diffusion rate of PNAN across the external surface into the internal pores of the adsorbents.

However, for NB-adsorbents systems, the adsorption performances were observed to be not favored by a rise in temperature. And the drop in the adsorption performances as the temperature rises could be due to exothermic adsorption. At the temperatures of $298 \mathrm{~K}, 308 \mathrm{~K}, 318 \mathrm{~K}$ and $328 \mathrm{~K}, \mathrm{NB}$ sorbed by MgFZBFA is $19.1,18.0,17.2$, and $16.0 \mathrm{mg} \mathrm{g}^{-1}$, respectively; and the amount sorbed by MgMZBFA is 8.1, $7.2,6.0$, and $5.1 \mathrm{mg} \mathrm{g}^{-1}$, respectively.

\section{Adsorption isotherms}

The $R^{2}$ values of the isotherm plots (Figs. 14 and 15) are serving as the measures of conformity of the adsorption processes with the assumptions of the theoretical adsorption isotherm. The Langmuir adsorption isotherm model better describes the adsorption processes involving PNAN and the adsorbents; and NB and the adsorbents. This is because the $R^{2}$ values obtained from the Langmuir isotherm plots (Table 4) for the adsorption processes are much closer to unity than the $R^{2}$ values obtained from the corresponding Freundlich adsorption isotherm plots (Table 5). This suggests the dominance of monolayer adsorption. Also, the values of the Langmuir coefficients $R_{\mathrm{L}}$ for all the adsorption processes are between 0 and 1 which suggests the favorability of the adsorption processes. And the values of the maximum monolayer sorption capacities $Q_{\mathrm{L}}$ (used as a measure of adsorption performance) of the adsorbents MgFZBFA and MgMZBFA for the sorption of the pollutants PNAN and NB (Table 4) show that, for the sorption of the two pollutants, $Q_{\mathrm{L}}(\mathrm{MgFZBFA})>Q_{\mathrm{L}}(\mathrm{MgMZBFA})$.

The aforementioned trend for the $Q_{\mathrm{L}}$ has relationships with some of the physical properties determined for the adsorbents. It corresponds to the surface area and porosity trend. MgFZBFA with the higher set of $Q_{\mathrm{L}}$ values for PNAN and NB has the larger surface area $\left(83.02 \mathrm{~m}^{2} \mathrm{~g}^{-1}\right)$ and pore volume $\left(0.29 \mathrm{~cm}^{3} \mathrm{~g}^{-1}\right)$, while MgMZBFA with
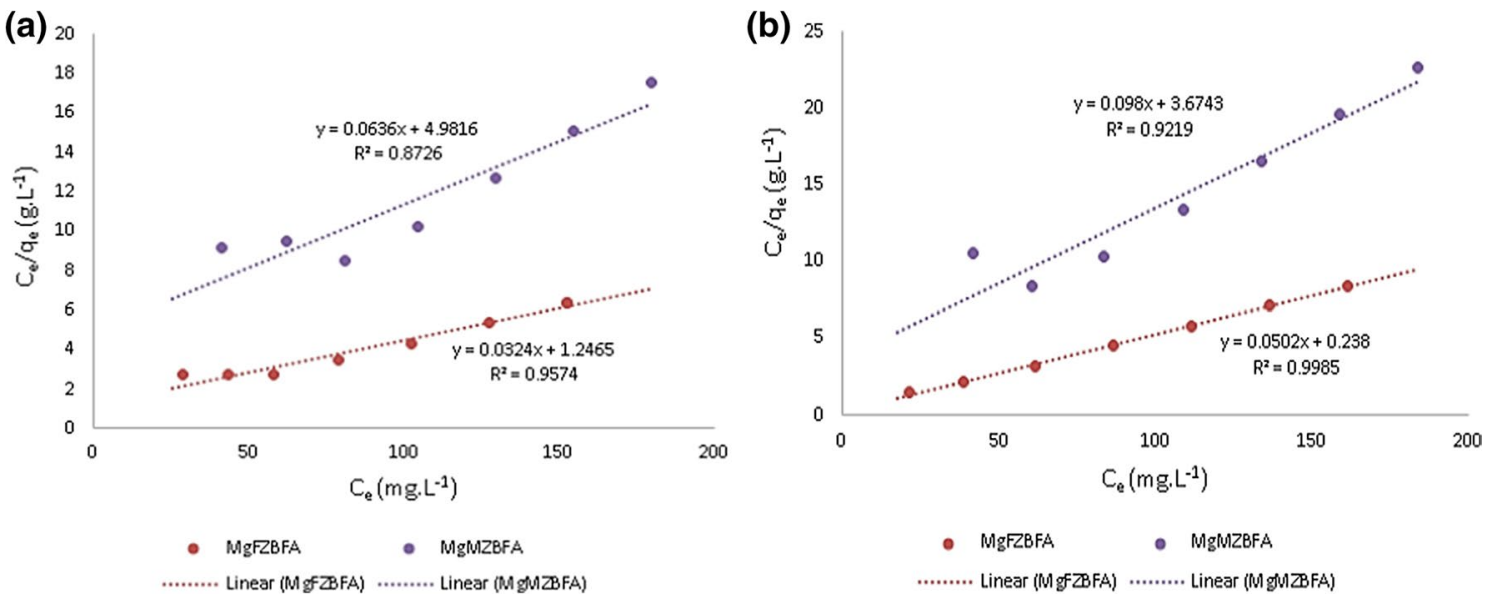

Fig. 14 Langmuir isotherm plots for the sorption of a PNAN, b NB on MgFZBFA and MgMZBFA 

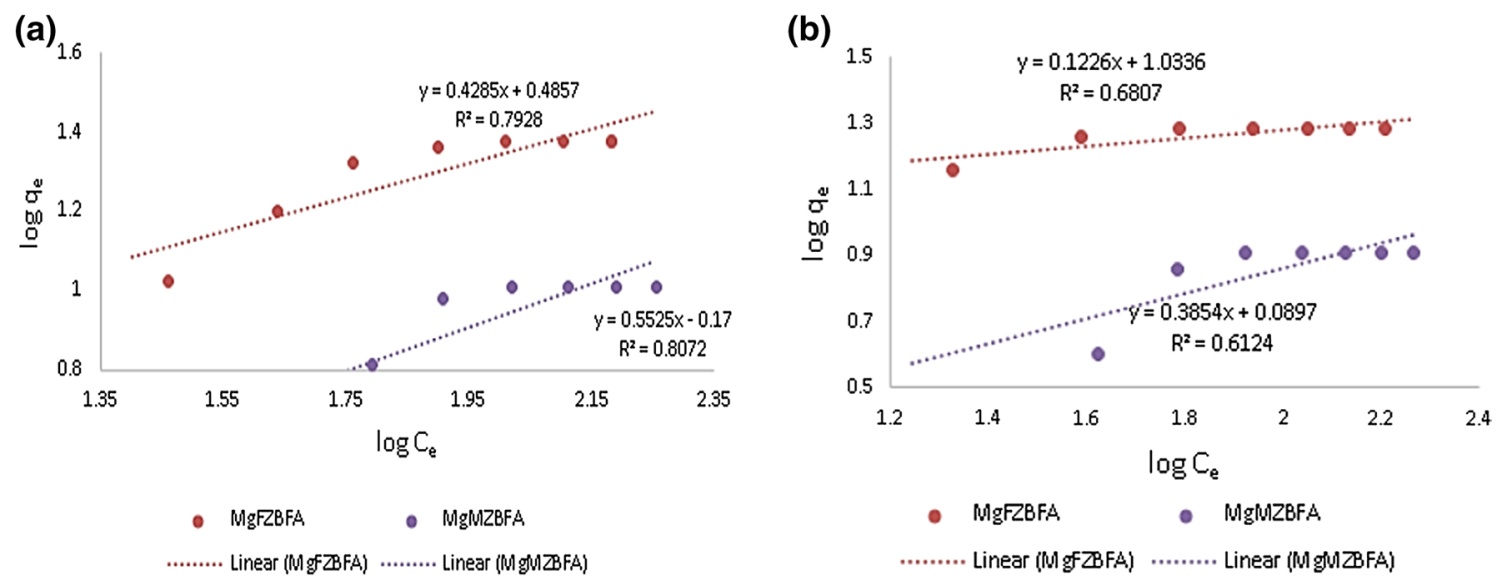

Fig. 15 Freundlich isotherm plots for the sorption of a PNAN b NB on MgFZBFA and MgMZBFA

Table 4 Langmuir isotherm parameters for the sorption of PNAN and $\mathrm{NB}$ on MgFZBFA and MgMZBFA

\begin{tabular}{llllll}
\hline Adsorbate & Sorbents & \multicolumn{4}{l}{ Parameter values } \\
\cline { 3 - 6 } & & $\begin{array}{l}Q_{\mathrm{L}} \\
\left(\mathrm{mg} \mathrm{g}^{-1}\right)\end{array}$ & $\begin{array}{l}K_{\mathrm{L}} \\
\left(\mathrm{mg} \mathrm{g}^{-1}\right)\end{array}$ & $\begin{array}{l}R_{\mathrm{L}} \\
\left(\mathrm{L} \mathrm{mg}^{-1}\right)\end{array}$ & $R^{2}$ \\
\hline PNAN & MgFZBFA & 30.86 & 0.026 & 0.277 & 0.9574 \\
& $\begin{array}{l}\text { MgMZ- } \\
\text { BFA }\end{array}$ & 12.72 & 0.013 & 0.438 & 0.8726 \\
NB & MgFZBFA & 19.92 & 0.211 & 0.045 & 0.9985 \\
& MgMZ- & 10.20 & 0.027 & 0.271 & 0.9219 \\
& BFA & & & & \\
\hline
\end{tabular}

Table 5 Freundlich isotherm parameters for the sorption of PNAN and NB on MgFZBFA and MgMZBFA

\begin{tabular}{lllll}
\hline Adsorbate & Sorbents & \multicolumn{4}{l}{ Parameter values } \\
\cline { 3 - 5 } & & $K_{\mathrm{F}}$ & $n$ & $R^{2}$ \\
\hline PNAN & MgFZBFA & 3.060 & 2.334 & 0.7928 \\
& MgMZBFA & 0.676 & 1.810 & 0.8072 \\
NB & MgFZBFA & 10.804 & 8.157 & 0.6807 \\
& MgMZBFA & 1.229 & 2.595 & 0.6124 \\
\hline
\end{tabular}

the lower set of $Q_{\mathrm{L}}$ values for the sorption of PNAN and NB has the lower surface area $\left(3.26 \mathrm{~m}^{2} \mathrm{~g}^{-1}\right)$ and pore volume $\left(0.01 \mathrm{~cm}^{3} \mathrm{~g}^{-1}\right)$. Also, the density of the adsorbents (whose increase in magnitude from one adsorbent to the other has an inverse relationship with their porous properties), 0.33 and 0.69 for MgFZBFA and MgMZBFA, respectively, has an inverse trend with the sets of $Q_{\mathrm{L}}$ values of the adsorbents for the adsorption of the PNAN and NB. All of these suggest that the variation in these physical properties most likely have an influence on the adsorption capacities of the adsorbents for the pollutants.
Table 6 Comparison of the maximum adsorption capacities of sorbents for PNAN and NB

\begin{tabular}{llll}
\hline Adsorbate & Sorbent & $Q_{\mathrm{L}}\left(\mathrm{mg} \mathrm{g}^{-1}\right)$ & Reference \\
\hline PNAN & Cliniptilolit & 0.85 & Ma et al. (2007) \\
& Humic acid & 1.8 & Wu et al. (2007) \\
& MgFZBFA & 30.86 & This work \\
& MgMZBFA & 12.72 & This work \\
NB & Bentonite & 10.8 & Gzhu et al. (1997) \\
& Active carbon & 2.4 & Chena et al. (2009) \\
& Nanocrystalline & 0.8 & Wei et al. (2010) \\
hydroxyapatite & & \\
& Hydroxyapa- & 7.32 & Weia et al. (2014) \\
tite-gelatin & & \\
nanocomposite & & This work \\
& MgFZBFA & 19.92 & This work \\
\hline
\end{tabular}

The maximum adsorption capacities $\left(Q_{\mathrm{L}}\right)$ of MgFZBFA and MgMZBFA compared to some other adsorbents previously studied for the removal of PNAN and NB (Table 6) show that the adsorbents of the present study manifested improved performances.

\section{Sorption dynamics}

At the optimal sorption conditions, the uptake of PNAN (100 $\mathrm{mg} \mathrm{L}^{-1}$, $\mathrm{pH} 4$ and $2.0 \mathrm{~g} \mathrm{~L}^{-1}$ adsorbents dose) and NB (100 $\mathrm{mg} \mathrm{L}^{-1}$, pH 9 and $2.0 \mathrm{~g} \mathrm{~L}^{-1}$ adsorbents dose) from their respective aqueous solutions by the adsorbents MgFZBFA and MgMZBFA increased with equilibration time until about $2 \mathrm{~h}$, when the adsorbents surfaces seem saturated, and beyond, there seems to be no significant uptake of the pollutants.

The equations of the linear graphs (Figs. 16 and 17) from which the kinetic parameters for the pseudo-first-order 

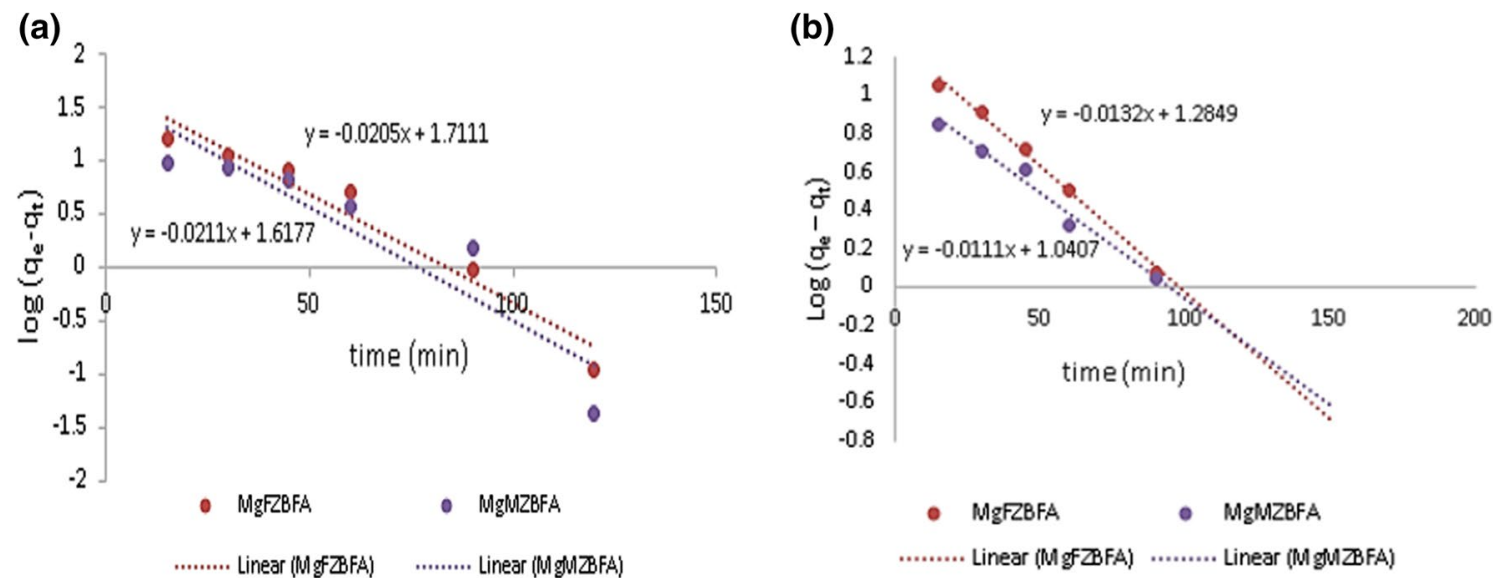

Fig. 16 Pseudo-first-order kinetic model plots for the sorption of a PNAN, b NB on MgFZBFA and MgMZBFA
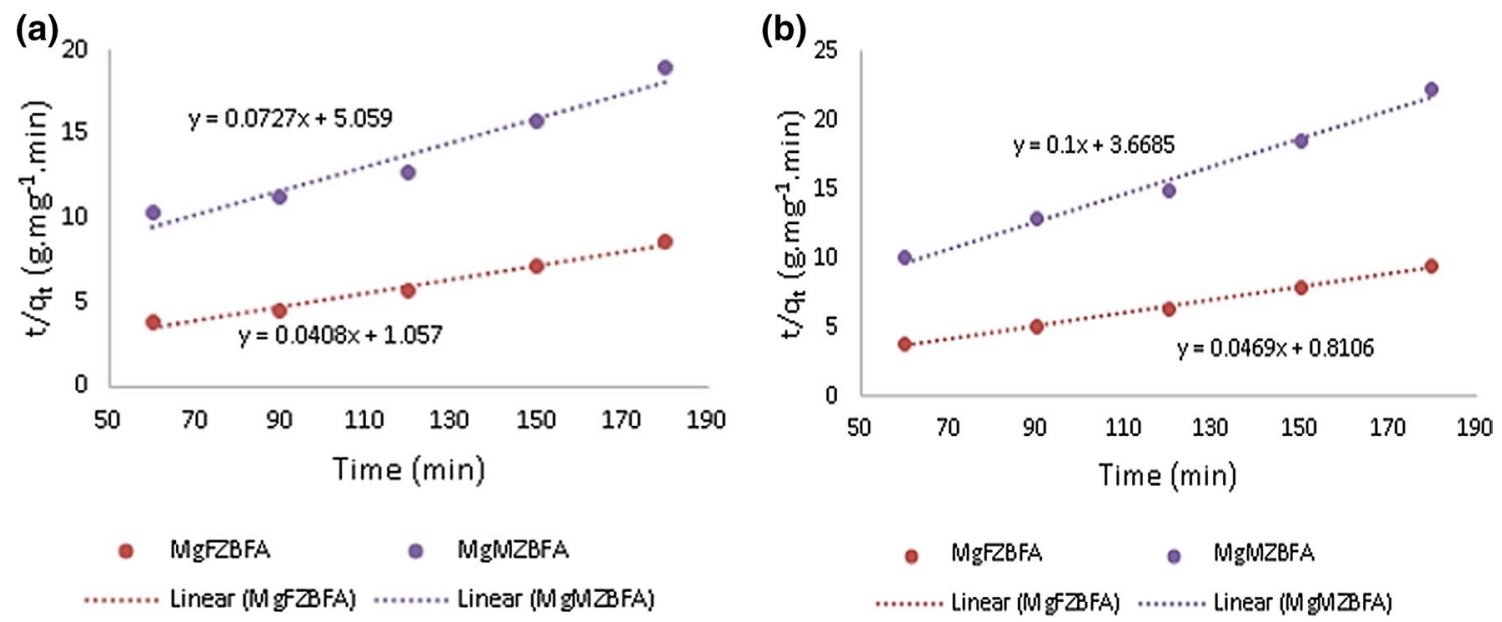

Fig. 17 Pseudo-second-order kinetic model plots for the sorption of a PNAN, b NB on MgFZBFA and MgMZBFA

Table 7 Pseudo-first-order kinetic model parameters for the sorption of PNAN and NB on MgFZBFA and MgMZBFA

\begin{tabular}{lllll}
\hline Adsorbate & Sorbent & \multicolumn{3}{l}{ Kinetic parameter values } \\
\cline { 3 - 5 } & & $k_{1}\left(\mathrm{~min}^{-1}\right)$ & $q_{\mathrm{e}}\left(\mathrm{mg} \mathrm{g}^{-1}\right)$ & $R^{2}$ \\
\hline PNAN & MgFZBFA & 0.047 & 51.42 & 0.9515 \\
& MgMZBFA & 0.049 & 41.47 & 0.8504 \\
NB & MgFZBFA & 0.030 & 19.27 & 0.9946 \\
& MgMZBFA & 0.026 & 10.98 & 0.9801 \\
\hline
\end{tabular}

kinetic model (Table 7) and pseudo-second-order kinetic model (Table 7) for the sorption processes involving the adsorbents MgFZBFA and MgMZBFA, and the pollutants PNAN and NB were obtained are included in the kinetic modelling plots for the adsorption processes.

The $R^{2}$ values of the kinetics models plots (Tables 7 and 8 ) are serving as the measures of conformity of the adsorption processes with the assumptions of the theoretical adsorption kinetics models. The pseudo-second-order kinetic model better fits the adsorption processes involving
Table 8 Pseudo-second-order kinetic model parameters for the sorption of PNAN and NB on MgFZBFA and MgMZBFA

\begin{tabular}{llllll}
\hline Adsorbate & Sorbent & \multicolumn{2}{l}{ Kinetic parameter values } \\
\cline { 3 - 5 } & & $k_{2}\left(\mathrm{~g} \mathrm{mg}^{-1} \mathrm{~min}^{-1}\right)$ & $q_{\mathrm{e}}\left(\mathrm{mg} \mathrm{g}^{-1}\right)$ & $h_{\mathrm{o}}\left(\mathrm{mg} \mathrm{g}^{-1} \mathrm{~min}^{-1}\right)$ & $R^{2}$ \\
\hline PNAN & MgFZBFA & $1.58 \times 10^{-3}$ & 24.51 & 0.95 & 0.9859 \\
& MgMZBFA & $1.05 \times 10^{-3}$ & 13.75 & 0.20 & 0.9447 \\
NB & MgFZBFA & $2.71 \times 10^{-3}$ & 21.32 & 1.23 & 0.9966 \\
& MgMZBFA & $2.73 \times 10^{-2}$ & 10.01 & 0.27 & 0.9868 \\
\hline
\end{tabular}


PNAN and the adsorbents; and NB and the adsorbents because the $R^{2}$ values obtained from the pseudo-secondorder kinetic model plots for the adsorption processes are much closer to unity than the $R^{2}$ values obtained from the corresponding pseudo-first-order kinetic model plots for the adsorption processes. Hence, the sorption processes could be said to predominantly involve the interaction of the adsorbates with the surfaces of the adsorbents, and most likely is the rate-determining step.

The intra-particle diffusion plots for all the adsorption processes (Fig. 18) show two linear sections, and this implies that the adsorption processes were most possibly completed by both surface sorption (liquid-film diffusion) and intra-particle diffusion (Lorenc-Grabowska and Gryglewicz 2007). The surface sorption is represented by the first (left) linear sections of the plots, while the intra-particle diffusion which involved the inward movement of the pollutants through the pores of the adsorbents, is represented by the second (right) linear sections of the plots. The rate constant of pore diffusion $\left(k_{\mathrm{i}}\right)$ characterizes the rate of the adsorption processes in this region (second linear portions of the plots) where pore diffusion is rate determining. The values for $k_{\mathrm{i}}$ for each adsorption processes (Table 9) were determined from the slope of the second linear portions of the plots (Sarkar et al. 2003). The intercepts of the plots, on the other hand, signifies the extent of the boundary layer effect $(I)$ for the adsorption processes (Table 8). The larger the boundary layer, the greater the contribution of surface adsorption as the rate determining step of an adsorption process.

Therefore, the mechanisms of the adsorption processes involving the pollutants PNAN and NB in their respective aqueous solutions with the adsorbents MgFZBFA and MgMZBFA most likely involves: the mass movement of the aqueous solutions of PNAN and NB toward the solid phase boundaries of the adsorbents; the transport of PNAN and NB from the solutions to the surface of the adsorbents and their
Table 9 Intraparticle diffusion kinetic model parameters for the sorption of PNAN and NB on MgFZBFA and MgMZBFA

\begin{tabular}{lllll}
\hline Adsorbate & Sorbent & \multicolumn{4}{l}{ Kinetic parameter values } \\
\cline { 3 - 5 } & & $\begin{array}{l}k_{\mathrm{i}} \\
\left(\mathrm{mg} \mathrm{g}^{-1} \mathrm{~min}^{-1 / 2}\right)\end{array}$ & $I\left(\mathrm{mg} \mathrm{g}^{-1}\right)$ & $R^{2}$ \\
\hline PNAN & MgFZBFA & 0.227 & 18.11 & 0.7393 \\
& MgMZBFA & 0.358 & 5.00 & 0.6686 \\
NB & MgFZBFA & 0.284 & 15.62 & 0.6491 \\
& MgMZBFA & 0.265 & 4.78 & 0.6492 \\
\hline
\end{tabular}

interactions with the adsorption sites; and the inward diffusion of the pollutants through the pores of the adsorbents. And the interaction of the pollutants with the surface of the adsorbents being the rate determining step in the adsorption processes.

\section{Regeneration and reusability of the adsorbents}

During PNAN desorption, hydrochloric acid brought down the $\mathrm{pH}$ of the adsorption system to that which favors the conversion of the adsorbate species to their respective cationic forms due to the interaction with hydrogen ions, hence becoming more dispersible into the aqueous phase. Lower desorption of NB with mineral acids and sodium hydroxide compared with acetic acid was reported by Rauthula and Srivastava. However, acetic acid performed best (Rauthula and Srivastava 2011) being organic and having the tendency to undergo hydrogen bonding interaction with $\mathrm{NB}$, hence fostering the transfer of NB into the liquid phase.

Three rounds of the PNAN and NB adsorption measurements were carried out on the adsorbents, with the final two coming after each recovery stage. Results (Figs. 19 and 20) show that the adsorbents were sorption active for the three rounds. The sorption capability of the adsorbents $q_{\mathrm{e}}$ were
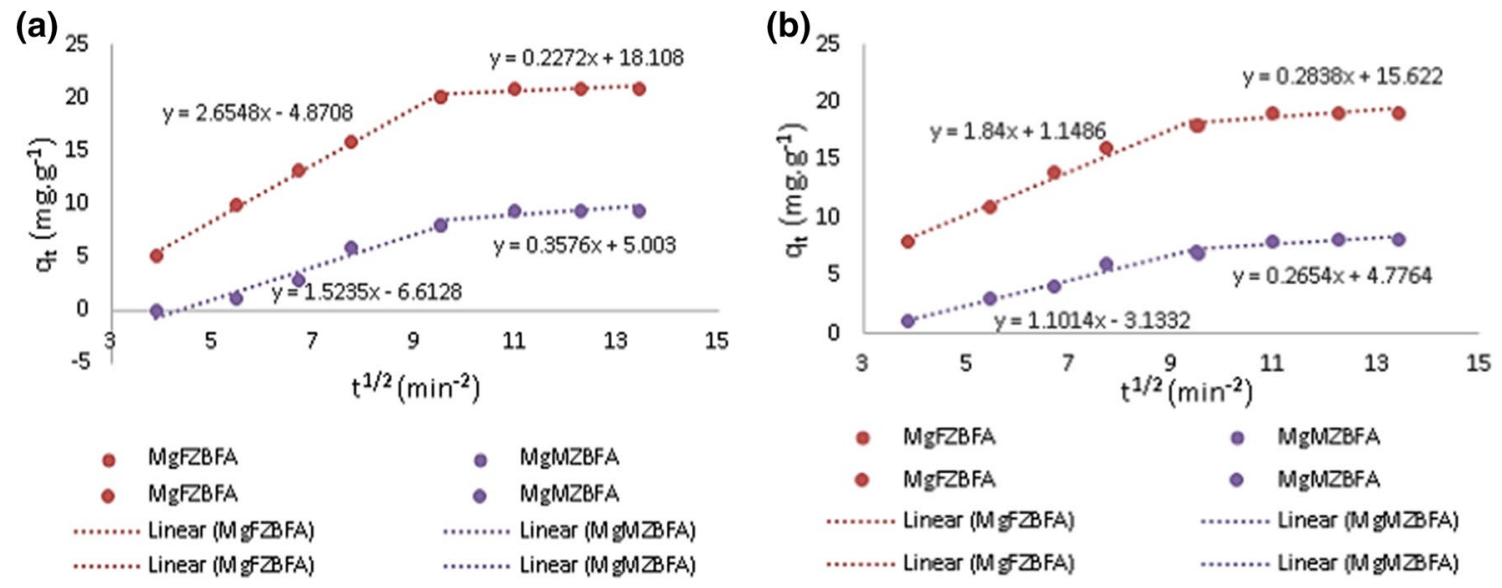

Fig. 18 Intraparticle diffusion model plots for the adsorption of a PNAN, b NB on MgFZBFA and MgMZBFA 


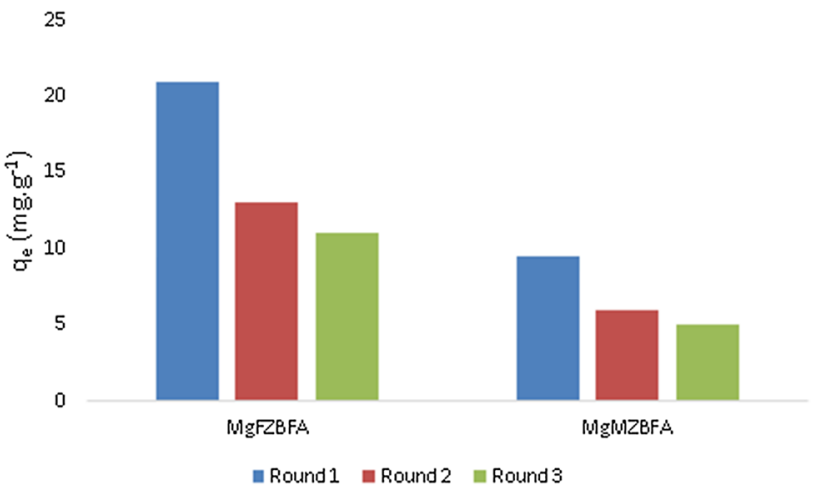

Fig. 19 Regeneration (from PNAN sorption) and reuse of MgFZBFA and MgMZBFA

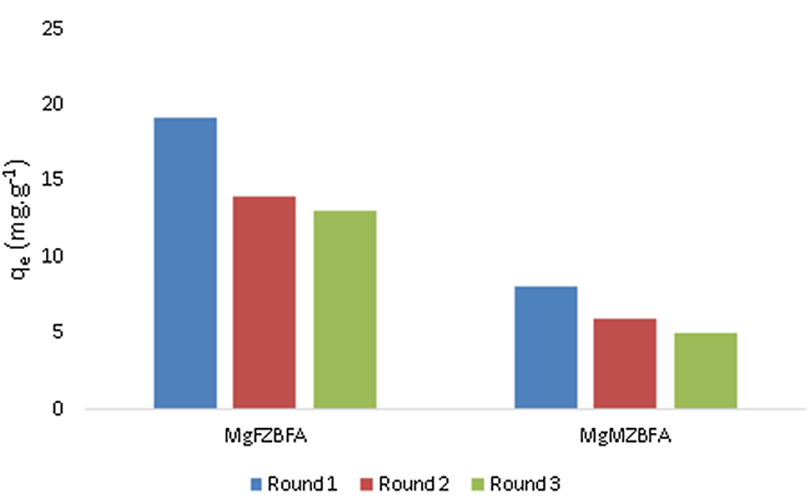

Fig. 20 Regeneration (from NB sorption) and reuse of MgFZBFA and MgMZBFA

observed to dwindle, most notably after the first regeneration. This suggests that the adsorbents were not fully recovered from the adsorbates due to chemisorption that might have played a role alongside physisorption in the adsorption processes. Also, acid wash might have brought about some of the structural defects in the zeolitic structure and might have led to the leach out of some $\mathrm{Mg}$ (II) ions in the structure which in turn might have caused decreased active sites of the adsorbent. However, the $q_{\mathrm{e}}$ values of the adsorbent did not reduce below half of their respective initial $q_{\mathrm{e}}$ after the third round of adsorption-desorption cycle.

\section{Conclusions}

Bagasse fly ash, a sugar industry solid waste was successfully transformed into mesoporous composite zeolitic sorbents MgFZBFA and MgMZBFA. After consideration of the results obtained from sorption studies, both sorbents possess the ability to remove PNAN and NB from aqueous solutions. However, MgFZBFA, which is a product of alkali fusion synthesis sorbed the pollutants better than MgMZBFA, a product of microwave alkaline hydrothermal synthesis. This is due to the higher surface area, more porous nature, and the higher amount of charge sites of the former (caused by better inclusion of aluminum and magnesium during synthesis). The sorptive ability of these sorbents assigns to them the potential of being applied as an economic means of removing PNAN, NB, and possibly some other pollutants from contaminated water.

Acknowledgements The authors acknowledge Shree Khedut Sahkari Khand Udhyog Mandali Ltd., Bardoli, India for providing the bagasse fly ash which was used for this research. One of the authors OAO acknowledges the Indian Council for Cultural Relations (ICCR), New Delhi for providing a scholarship. The authors acknowledge the reviewers for their precious contributions toward improving this article.

Funding The author(s) received no specific funding for this work.

\section{Compliance with ethical standards}

Conflict of interest The authors hereby declare that there is no conflict of interests regarding the publication of this article.

Open Access This article is licensed under a Creative Commons Attribution 4.0 International License, which permits use, sharing, adaptation, distribution and reproduction in any medium or format, as long as you give appropriate credit to the original author(s) and the source, provide a link to the Creative Commons licence, and indicate if changes were made. The images or other third party material in this article are included in the article's Creative Commons licence, unless indicated otherwise in a credit line to the material. If material is not included in the article's Creative Commons licence and your intended use is not permitted by statutory regulation or exceeds the permitted use, you will need to obtain permission directly from the copyright holder. To view a copy of this licence, visit http://creativecommons.org/licenses/by/4.0/.

\section{References}

Amodu OS, Ojumu TV, Ntwampe SK, Ayanda OS (2015) Rapid adsorption of crystal violet onto magnetic zeolite synthesized from fly ash and magnetite nanoparticles. J Encapsul Adsorpt Sci 5:191-203

Bhatkhande DS, Kamble SP, Sawant SB, Pangarkar VG (2004) Photocatalytic and photochemical degradation of nitrobenzene using artificial ultraviolet light. Chem Eng J 102:283-290. https://doi. org/10.1016/j.cej.2004.05.009

Cardenas-Pena AM, Ibanez JG, Vasquez-Medrano R (2012) Determination of the point of zero charge for electrocoagulation precipitates from an iron anode. Int J Electrochem Sci 7:6142-6153

Chena M, Cuia L, Li C, Diao G (2009) Adsorption, desorption and condensation of nitrobenzene solution from active carbon: a comparison of two cyclodextrins and two surfactants. J Hazard Mater 162:23-28

Dias NC, Steiner PA, Braga MCB (2015) Characterization and modification of a clay mineral used in adsorption tests. J Miner Mater Charact Eng 3:277-288

EPA Nitrobenzene. https://www.epa.gov/sites/production/files/201609/documents/nitrobenzene.pdf. Accessed 8 May 2018 
Fiol N, Villaescusa I (2009) Determination of sorbent point of zero charge: usefulness in sorption studies. Environ Chem Lett 7:7984. https://doi.org/10.1007/s10311-008-0139-0

Gzhu L, Li Y, Zhang J (1997) Sorption of organobentonites to some organic pollutants in water. Environ Sci Technol 31:1407-1410

Hildebrando EA, Andrade CGB, Junior CAFR, Angélica RS, Valenzuela-Diaz FR, Neves RF (2014) Synthesis and characterization of zeolite $\mathrm{NaP}$ using kaolin waste as a source of silicon and aluminum. Mate Res 17:174-179

Huang TY, Chuieh PT (2015) Life cycle assessment of reusing fly ash from municipal solid waste incineration. Procedia Eng 118:984-991

James J, Pandian PK (2017) A short review on the valorisation of sugarcane bagasse fly ash in the manufacture of stabilized/sintered earth blocks and tiles. Adv Mater Sci Eng 2017:1-15. https://doi. org/10.1155/2017/1706893

Jha B, Singh DN (2016) Flyash zeolites: innovations, applications, and directions. Adv Struct Mater 78:5-31. https://doi. org/10.1007/978-981-10-1404-8

Kalavathy MK, Karthikeyan T, Rajgopal S, Miranda LR (2005) Kinetic and isotherm studies of $\mathrm{Cu}(\mathrm{II})$ adsorption onto $\mathrm{H}_{3} \mathrm{PO}_{4}$-activated rubber wood sawdust. J Colloid Interface Sci 292:354-362. https ://doi.org/10.1016/j.jcis.2005.05.087

Lai $C$ et al (2018) Simultaneous degradation of P-nitroaniline and electricity generation by using a microfiltration membrane dual-chamber microbial fuel cell. Int J Hydrog Energy 43(3):1749-1757. https://doi.org/10.1016/j.ijhydene.2017.11.025

Li K, Lib Y, Zheng Z (2010) Kinetics and mechanism studies of p-nitroaniline adsorption on activated carbon fibers prepared from cotton stalk by NH4H2PO4 activation and subsequent gasification with steam. J Hazard Mater 178:553-559. https://doi. org/10.1016/j.jhazmat.2010.01.120

Liu Y (2008) New insights into pseudo-second-order kinetic equation for adsorption. Colloid Surf A 320:275-278. https://doi. org/10.1016/j.colsurfa.2008.01.032

Lorenc-Grabowska E, Gryglewicz G (2007) Adsorption characteristics of Congo Red on coal-based mesoporous activated carbon. Dyes Pigm 74:34-40. https://doi.org/10.1016/j.dyepig.2006.01.027

Ma MG, Wei YX, Zhang Y (2007) Modify of clinoptilolit (from Baiyin) and its adsorption of p-nitroaniline. J Anhui Agri Sci (Chinese) 35:2061-2062

Mahmood T, Saddique MT, Naeem A, Westerhoff P, Mustafa S, Alum A (2011) Comparison of different methods for the point of zero charge determination of NiO. Ind Eng Chem Res 50:1001710023. https://doi.org/10.1021/ie200271d

Majchrzak-Kuceba I (2013) A simple thermogravimetric method for the evaluation of the degree of fly ash conversion into zeolite material. J Porous Mater 20:407-415. https://doi.org/10.1007/ s10934-012-9610-1

Martison E (2014) EPA coal ash rule still not done. POLITICO. https ://www.politico.com/story/2014/03/epa-coal-ash-rule-104967. Accessed 26 Jan 2018

Moshoeshoe M, Nadiye-Tabbiruka MS, Obuseng V (2017) A review of the chemistry, structure, properties and applications of zeolites. Am J Mater Sci 7:196-221. https://doi.org/10.5923/j.mater ials.20170705.12

Musyoka NM, Petrik LF, Fatoba OO, Hums E (2013) Synthesis of zeolites from coal fly ash using mine waters. Miner Eng 53:9-15. https://doi.org/10.1016/j.mineng.2013.06.019

Nair RS, Johannsen FR, Levinskas GJ, Terrill JB (1986) Subchronic inhalation toxicity of p-nitroaniline and p-nitrochlorobenzene in rats. Fundam Appl Toxicol 6:618-627

Nishino SF, Spain JC (1993) Degradation of nitrobenzene by a Pseudomonas pseudoalcaligenes. Appl Environ Microbiol 59:2520-2525
NJDEP. Hazardous substance fact sheet. New Jersey Department of Health and Senior Services. http://nj.gov/health/eoh/rtkweb/ documents/fs/1548.pdf. Accessed 12 May 2018

Pan J, Guan B (2010) Adsorption of nitrobenzene from aqueous solution on activated sludge modified by cetyltrimethylammonium bromide. J Hazard Mater 183:341-346. https://doi.org/10.1016/j. jhazmat.2010.07.030

PubChem Nitrobenzene. https://pubchem.ncbi.nlm.nih.gov/compound/ nitrobenzene\#section=Analytic-Laboratory-Methods. Accessed 9 May 2018

Qin Q, Ma J, Liu K (2007) Adsorption of nitrobenzene from aqueous solution by MCM-41. J Colloid Interface Sci 315:80-86. https:// doi.org/10.1016/j.jcis.2007.06.060

Querol X, Moreno N, Umana JC, Alastuey A, Hernandez E, LopezSoler A, Plana F (2002) Synthesis of zeolites from coal fly ash: an overview. Int J Coal Geol 50:413-423. https://doi. org/10.1016/S0166-5162(02)00124-6

Raleigh NC (2016) Duke energy corporation agrees to $\$ 6$ million fine for coal ash spill, North Carolina says. CBS News. https://www. cbsnews.com/news/duke-energy-corporation-agrees-6-millionfine-coal-ash-spill-north-carolina. Accessed 26 Jan 2018

Rauf N, Damayanti MC, Pratama SWI (2017) The influence of sugarcane bagasse ash as fly ash on cement quality. In: AIP. https ://doi.org/10.1063/1.4973098

Rauthula MS, Srivastava VC (2011) Studies on adsorption/desorption of nitrobenzene and humic acid onto/from activated carbon. Chem Eng J 168:35-43. https://doi.org/10.1016/j. cej.2010.12.026

Sarkar M, Acharya PK, Bhattacharya B (2003) Modeling the adsorption kinetics of some priority organic pollutants in water from diffusion and activation energy parameters. J Colloid Interface Sci 266:28-32. https://doi.org/10.1016/S0021-9797(03)00551-4

Scott MA, Kathleen AC, Prabir KD (2003) Handbook of zeolite science and technology. Marcel Dekker Inc, New York

Shah BA, Pandya DD, Shah HA (2016) Impounding of ortho-chlorophenol by zeolitic materials adapted from bagasse fly ash: four factor three level box-behnken design modelling and optimization. Arab J Sci Eng 42:241-260. https://doi.org/10.1007/s1336 9-016-2294-0

Shah BA, Oluyinka OA, Shah AV (2019) Fly ash reuse as mesoporous $\mathrm{Ca}$ - and $\mathrm{Mg}$-zeolitic composites for the seclusion of aniline from aqueous solution. Arab J Sci Eng 44:289-304. https://doi. org/10.1007/s13369-018-3596-1

Sun J-H, Suna S-P, Fan M-H, Guoa H-Q, Qiao L-P, Sun R-X (2007) A kinetic study on the degradation of p-nitroaniline by Fenton oxidation process. J Hazard Mater 148:172-177. https://doi. org/10.1016/j.jhazmat.2007.02.022

Tanaka H, Furusawa S, Hino R (2002) Synthesis, characterization, and formation process of Na-X zeolite from coal fly ash. J Mate Synth Process 10:143-148. https://doi.org/10.1023/A:1021938729996

Tantawy MA, El-Roudi AM, Salem AA (2014) Utilization of bagasse ash as supplementary cementitious material. Int J Eng Res Technol 3:1342-1348

Tran HN, Wang Y, You S, Chao H (2017) Insights into the mechanism of cationic dye adsorption on activated charcoal: the importance of pie-pie interactions. Process Saf Environ Prot 107:168-180

Usachev NY, Belanova EP, IKrukovsky IM, Kanaev SA, Atal'yan OK, Kazakov AV (2003) Thermal transformations in systems based on zeolites Y, X, and A containing zinc and sodium nitrates. Russ Chem Bull, Int Ed 52:1940-1949

UW Adsorption Equilibria Principles. http://mimoza.marmara.edu. tr/ zehra.can/ENVE401/3.\%20Adsorption\%20Equilibria.pdf. Accessed 2 Feb 2018

Wang SB, Peng YL (2010) Natural zeolites as effective adsorbents in water and wastewater treatment. Chem Eng J 156:11-24. https:// doi.org/10.1016/j.cej.2009.10.029

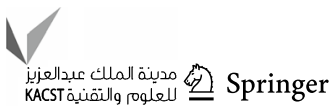


Weber WJ Jr, Morris JC (1963) Kinetics of adsorption on carbon from solution. J Sanit Eng Div Am Soc Civ Eng 89:31-60

Wei W, Sun R, Cui J, Wei Z (2010) Removal of nitrobenzene from aqueous solution by adsorption on nanocrystalline hydroxyapatite. Desalination 263:89-96. https://doi.org/10.1016/j.desal .2010 .06 .043

Weia W, Sunb R, Jina Z, Cuia J, Weia Z (2014) Hydroxyapatite-gelatin nanocomposite as a novel adsorbent for nitrobenzene removal from aqueous solution. Appl Surf Sci 292:1020-1029

Wu YQ, Zhou M, Ma MG, Zhang Y, Chen H (2007) Adsorption kinetics of p-nitroaniline on the insolubilized humic acid. Tec Water $\operatorname{Tr}$ (Chinese) 33:14-17

Wu DY, Lu YK, Kong HN, Ye C, Jin XC (2008) Synthesis of zeolite from thermally treated sediment. Ind Eng Chem Res 47:295-302. https://doi.org/10.1021/ie071063u
Xu R, Pang W, Yu J, Huo O, Chen J (2007) Chemistry of zeolites and related porous materials: synthesis and structure. Wiley, Singapore

Zheng K et al (2007) Enhanced adsorption of p-nitroaniline from water by a carboxylated polymeric adsorbent. Sep Purif Technol 57:250-256. https://doi.org/10.1016/j.seppur.2007.04.017

Publisher's Note Springer Nature remains neutral with regard to jurisdictional claims in published maps and institutional affiliations. 